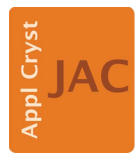

JOURNAL OF

APPLIED

CRYSTALLOGRAPHY

ISSN 1600-5767

\section{Characterization and application of Bragg-edge transmission imaging for strain measurement and crystallographic analysis on the IMAT beamline}

\author{
Ranggi S. Ramadhan, ${ }^{\mathrm{a}, \mathrm{b} *}$ Winfried Kockelmann, ${ }^{\mathrm{b}}$ Triestino Minniti, ${ }^{\mathrm{b}}$ Bo Chen, ${ }^{\mathrm{a}}$ \\ David Parfitt, ${ }^{a}$ Michael E. Fitzpatrick ${ }^{\mathrm{a}}$ and Anton S. Tremsin ${ }^{\mathrm{c}}$
}

${ }^{\mathbf{a}}$ Faculty of Engineering, Environment and Computing, Coventry University, Coventry CV1 2LD, UK, ${ }^{\mathbf{b}}$ ISIS Facility, Science
and Technology Facilities Council, Rutherford Appleton Laboratory, Chilton OX11 0QX, UK, and ${ }^{\mathbf{c}}$ Space Sciences
Laboratory, University of California, Berkeley, CA 94720, USA. *Correspondence e-mail: ramadhar@uni.coventry.ac.uk

Edited by G. Kostorz, ETH Zurich, Switzerland

Keywords: neutron imaging; Bragg edges; strain mapping; crystallographic texture mapping; IMAT.

Received 4 September 2018

Accepted 30 January 2019

This paper presents a series of experiments to characterize the performance of the new IMAT beamline at the ISIS pulsed neutron source and provides examples to showcase the potential applications of Bragg-edge transmission imaging on the instrument. The characterization includes determination of the IMAT spectral and spatial resolutions through calibration measurements, and also determination of the precision and the accuracy of Bragg-edge analysis for lattice parameters of ceramics, metals and textured engineering alloys through high-temperature measurements. A novel Bragg-edge analysis method based on the cross-correlation of different Bragg edges has been developed to provide an estimate of the change in lattice parameter, which is especially useful for measurements of textured samples. Three different applications of the Braggedge transmission imaging technique are presented, including strain mapping, texture mapping and obtaining crystallographic information, i.e. the dependence on temperature of the Debye-Waller factor. The experimental results demonstrate the ability of the IMAT beamline to provide accurate strain measurements with uncertainties as low as $90 \mu \varepsilon$ with reasonable measurement time, while characteristic materials parameters can be mapped across the sample with a spatial resolution of 300-600 $\mu \mathrm{m}$ for a strain map and down to $\sim 90 \mu \mathrm{m}$ for a texture map.

\section{Introduction}

Time-of-flight neutron transmission has emerged in recent years as a non-destructive technique for materials analysis, including texture and phase analysis (Sato et al., 2017; Sun et al., 2018; Woracek et al., 2015; Makowska et al., 2016), microstructure evaluation (Sato, Sato et al., 2015; Sato et al., 2011), strain mapping (Ramadhan et al., 2018; Tremsin, Ganguly et al., 2016; Tremsin, Yau \& Kockelmann, 2016; Strobl et al., 2012; Woracek et al., 2011), and strain tomography (Hendriks et al., 2017; Sato, Shiota et al., 2015; Abbey et al., 2012). Neutrons traversing crystalline materials produce sudden jumps in the transmitted intensity, known as Bragg edges, as a function of wavelength. The texture, phase and strain information in a material can be obtained through analysis of these Bragg edges (Santisteban et al., 2001; Vogel, 2000), and using a position-sensitive neutron detector system (Tremsin et al., 2015) these properties may be mapped across a bulk sample. This provides location-specific high-resolution microstructural information, averaged along the transmission direction. A technical review on the recent development of neutron transmission imaging techniques has been presented by Woracek et al. (2018). 
The versatility of this technique encouraged the development of the new imaging and materials science (IMAT) beamline at the ISIS pulsed neutron source, UK (Kockelmann et al., 2018). Recently, this beamline has gone through commissioning and it started its user programme in March 2018. The potential use of the IMAT beamline, ranging from engineering samples to archaeological objects, has been explored (Minniti et al., 2016), and a particular application of energy-selective neutron imaging to investigate biological samples on IMAT has been presented by Vitucci et al. (2018). The instrument parameters such as beam flux, beam profile and spectral resolution have been characterized (Minniti et al., 2018) without, however, evaluation of Bragg-edge transmission analysis on the instrument.

This paper presents the characterization of Bragg-edge transmission imaging on the IMAT beamline. The quality of the signal (in terms of statistical significance and sharpness) influences the accuracy of information derived from the Bragg edges (for example lattice parameters) and is different from one instrument to another. This instrumental effect is discussed in this paper, as are the achievable accuracy, uncertainty and spatial resolution of lattice-parameter determination on IMAT. Here, the definition of accuracy is the closeness of agreement to an actual value; uncertainty is the dispersion of the measured value; and spatial resolution is the smallest possible feature that can be detected from the reconstructed map. This paper also introduces a novel data analysis method to predict the lattice-parameter change in the presence of crystallographic texture. Texture changes the shape of the signal, and this often presents a significant challenge for Bragg-edge strain analysis. In this paper, example applications of Bragg edges for materials analysis are also presented. Residual strain mapping on an engineering sample is demonstrated. The use of Bragg-edge analysis beyond lattice-parameter determination is further highlighted by providing crystallographic information, such as crystallographic orientation and Debye-Waller factors.

While strain measurement and crystallographic analysis are well established subjects, the ability to map those parameters across a sample quickly and non-destructively is more challenging and is the main advantage of Bragg-edge neutron

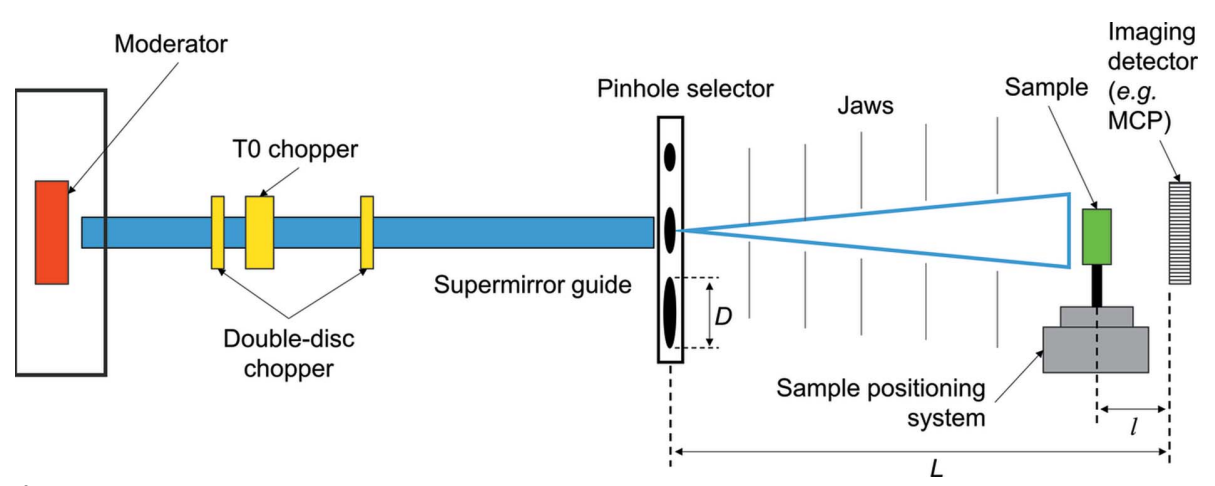

Figure 1

Outline of the IMAT instrument. The total flight path from moderator to sample is about $56 \mathrm{~m}$. The variable path $L$ from the pinhole to the imaging camera is about $10 \mathrm{~m}$. The sample is mounted on a seven-axis sample-positioning system. transmission imaging. This paper demonstrates the application of this technique to real engineering samples while also indicating the quality of the measurement results produced by the instrument. As such, this work summarizes essential results of the instrument characterization process, validates the results from Bragg-edge analysis on IMAT, and provides information and guidance on the design of future experiments as the beamline moves forward into the user programme.

\section{Basic concept}

\subsection{IMAT instrumentation}

IMAT is located at target station 2 (TS-2) of ISIS, and the outline of the instrument is shown in Fig. 1. A proton beam of $800 \mathrm{MeV}$ operating at $10 \mathrm{~Hz}$ bombards a tungsten target. Neutrons produced in the target are slowed down by a coupled $18 \mathrm{~K}$ hydrogen moderator and are transported to the pinhole selector through a $44 \mathrm{~m}$-long supermirror guide operating in vacuum. In the neutron guide, a $300 \mathrm{~mm}$-thick, $20 \mathrm{~Hz}$ T0 chopper as indicated in Fig. 1 is installed for fast neutron and gamma radiation filtering, using Inconel as the main absorbing material. Two $10 \mathrm{~Hz}$ double-disc choppers are installed to provide the wavelength band and to prevent overlap of neutrons between successive time frames. Several beam monitors are also installed along the guide. The pinhole selector located at $46 \mathrm{~m}$ from the moderator provides a variable aperture to define the beam divergence. There are five circular apertures, with diameters $D$ of $5,10,20,40$ and $80 \mathrm{~mm}$, and one square aperture of $100 \times 100 \mathrm{~mm}$ available on IMAT. From the aperture, the neutrons travel through $9 \mathrm{~m}$ vacuum tubes. Five sets of 'jaws' (or beam limiters) made of $10 \mathrm{~mm}$ thick $\mathrm{B}_{4} \mathrm{C}$ blades are integrated in the flight tubes to shape the size of the neutron beam as illustrated in Fig. 1. The neutrons eventually reach the sample position at $L=10 \mathrm{~m}$, with the beam divergence predominantly defined by the $L / D$ ratio. The beam divergence, together with sample-to-detector distance $l$, affects the geometrical blurring $d$ through the relation

$$
d=l /(L / D) .
$$

A microchannel plate (MCP) detector (Tremsin et al., 2013) is available on IMAT and currently used as the main time-of-flight (TOF) imaging camera. Neutrons are converted to electrons in a ${ }^{10} \mathrm{~B}$ - and $\mathrm{Gd}$ doped MCP. The charge is amplified by a second MCP and read out by a $2 \times 2$ array of Timepix chips. For each pixel, the neutron arrival time relative to the external trigger (provided by the pulsed neutron source) is registered and stored in a histogram with a maximum of about 3100 time channels, allowing energy-resolved measurement with the knowledge of the flightpath parameter. Each Timepix chip has $256 \times 25655 \times 55 \mu \mathrm{m}$ pixels, 
providing a sensitive area of $14 \times 14 \mathrm{~mm}$; i.e. the four chips provide a total field of view of $28 \times 28 \mathrm{~mm}$. A more detailed description of the IMAT instrument design and installation can be found elsewhere (Minniti et al., 2018).

\subsection{Principle of Bragg-edge transmission imaging}

Bragg edges occur as a result of the backscattering phenomenon where a set of lattice planes $(h k l)$ with lattice spacing $d_{h k l}$ is no longer contributing to neutron coherent scattering at a wavelength described by Bragg's law:

$$
\lambda=2 d_{h k l} \sin \theta,
$$

where $\lambda$ is the neutron wavelength and $\theta$ is the Bragg angle between the neutron beam and the lattice plane $\left(\theta=90^{\circ}\right.$ for backscattering). As for Bragg diffraction, crystallographic material parameters can be derived from these Bragg-edge signals, for example the lattice spacing and crystallographic orientations. Using a TOF imaging camera, these parameters can be mapped across the sample with the caveat that the information is averaged through the sample thickness in the beam-propagation direction.

Lattice spacings can be determined by the positions of the Bragg edges, which is analogous to Bragg-peak analysis in diffraction measurements. Fig. 2(a) compares Bragg edges and Bragg peaks for an aluminium alloy 2024 (A12024) powder. The Bragg edges, shown by blue symbols, were obtained on IMAT by measuring the powder sample inside an aluminium foil container with a dimension of $35 \mathrm{~mm}$ in the transmission direction for $4 \mathrm{~h}$. The same powder was measured on ENGIN-X, ISIS, in both diffraction mode and transmission mode using the MCP detector, shown by the black line and red symbols in Fig. 2(a), respectively. The powder was measured using a gauge volume of $2 \times 2 \times 2 \mathrm{~mm}$ for $0.5 \mathrm{~h}$ in diffraction mode. For measurement in transmission mode, the powder was inserted into a container with a dimension of $50 \mathrm{~mm}$ in the transmission direction and measured for $6 \mathrm{~h}$. In Fig. 2(a), the transmission intensity from IMAT and ENGIN-X and the normalized diffracted neutron counts are plotted as a function of $d$ spacing. As can be seen, the Bragg edges and Bragg peaks occur at the same lattice spacing of the first five $\mathrm{Al}$ reflections measured. The difference in intensity between the IMAT and ENGIN-X transmission signals is caused by the samplethickness difference in the transmission direction (i.e. the difference in transmission path lengths: $35 \mathrm{~mm}$ on IMAT and $50 \mathrm{~mm}$ on ENGIN-X). The gaps in the transmission signal, which are more notable in the ENGIN-X signal, are related to the data readout setting of the detector. The ENGIN-X transmission spectrum has a higher statistical error [Fig. 2(a)], despite being detected from the same detection-area size as the spectrum from IMAT $(160 \times 160$ pixels $)$ and integrated over a longer counting time $(6 \mathrm{~h}$, compared with $4 \mathrm{~h}$ on IMAT), demonstrating the higher beam flux at IMAT.

Precise determination of the lattice spacing from a Bragg edge may be achieved by using a nonlinear fit, for example with a function given by Santisteban et al. (2001) for TOF Bragg-edge fitting, which is shown in equation (3):

$$
\begin{aligned}
\operatorname{Tr}(\lambda) & =\exp \left[-\left(a_{0}+b_{0} \lambda\right)\right] \\
& \times\left(\exp \left[-\left(a_{h k l}+b_{h k l} \lambda\right)\right]+\left\{1-\exp \left[-\left(a_{h k l}+b_{h k l} \lambda\right)\right]\right\}\right) \\
& \times \frac{1}{2}\left[\operatorname{erfc}\left(-\frac{\lambda-\lambda_{h k l}}{2^{1 / 2} \sigma}\right)-\exp \left(-\frac{\lambda-\lambda_{h k l}}{\tau}+\frac{\sigma^{2}}{2 \tau^{2}}\right)\right. \\
& \left.\times \operatorname{erfc}\left(-\frac{\lambda-\lambda_{h k l}}{2^{1 / 2} \sigma}+\frac{\sigma}{\tau}\right)\right]
\end{aligned}
$$

where $\operatorname{Tr}(\lambda)$ is the transmission at neutron wavelength $\lambda$; constants $a_{0}$ and $b_{0}$ describe the exponential function on the right-hand side of the Bragg edge; $a_{h k l}$ and $b_{h k l}$ describe the jump in total neutron cross section, i.e. providing the Braggedge height information; $\lambda_{h k l}$ is the Bragg-edge position; $\tau$ is a decay constant which is an intrinsic property of the moderator; and $\sigma$ is the Gaussian broadening due to sample and instrument geometry. Fig. 2(b) shows a nonlinear least-squares fit of the function in equation (3) to the $\mathrm{Al}\{111\}$ Bragg edges. Despite the different shapes of the edges from the two different instruments, equation (3) yields almost identical $\{111\}$ lattice spacings of $2.3378 \pm 0.0006$ and $2.3382 \pm 0.0001 \AA$ for IMAT and ENGIN-X Bragg edges, respectively. The
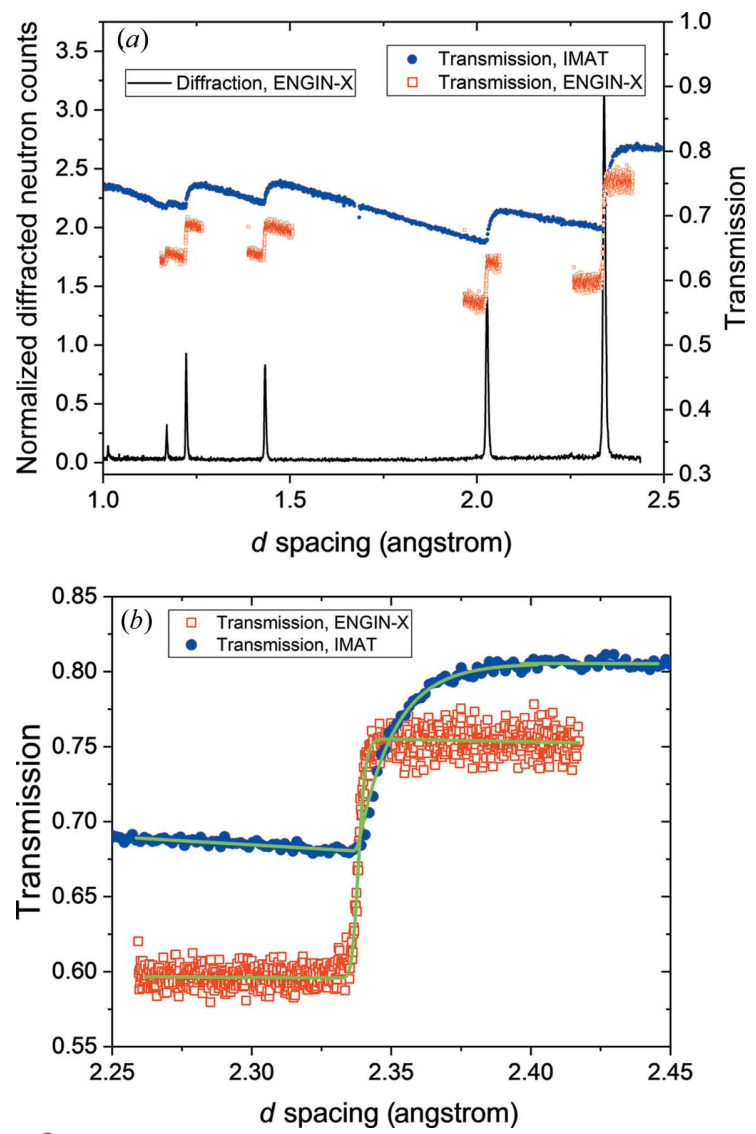

Figure 2

(a) Bragg edges and Bragg diffraction peaks of Al2024 powder fall on top of each other, providing the lattice-spacing information of the first five aluminium reflections measured. Bragg edges were measured on IMAT (blue symbols) and ENGIN-X (red symbols), and have different shapes owing to the different resolution functions of the two instruments. The diffraction pattern (black line) was measured on ENGIN-X. (b) A nonlinear function given in equation (3) (green lines) describes the Bragg edges from the two instruments well. 
difference is smaller than the fitting error and equivalent to $\sim 171 \mu \varepsilon$. In principle, the map of the lattice spacing, and also other parameters of equation (3), can be reconstructed pixel by pixel across the field of view. However, each individual pixel may not have enough neutron events within a reasonable measurement time to provide good counting statistics for fitting. Therefore, MCP pixels are usually combined to form larger regions ('macro-pixels'). A combined pixel bin of $10 \times$ 10 to $20 \times 20$ pixels is commonly used for analysis to improve the statistics, with the running average of the macro-pixel with step size of 1 pixel $(55 \mu \mathrm{m})$ applied across the image (Tremsin, Ganguly et al., 2016). The use of larger macro-pixels will consequently decrease the spatial resolution of the reconstructed map. The pixel-by-pixel fitting across the sample is automated using Bragg-edge mapping software such as BEATRIX (Minniti, 2019), which utilizes the seven-parameter equation (3), and TPX_Edgefit (Anton Tremsin, personal communication, 1 October 2016), which has a nonlinear fitting function with five parameters implemented (Tremsin et al., 2009), where the transmission before the Bragg edge and the Bragg-edge height are described with one single coefficient each. Despite having fewer parameters, the latter function works for fitting a narrow region of the transmission spectrum around the Bragg edge.

Crystallographic orientation mapping can be achieved using energy-selective transmission imaging (Kockelmann et al., 2007) for a specific neutron wavelength $\lambda$, to produce a map of the number of crystallites having any of the $(h k l)$ plane normals making an angle $\beta_{h k l}$ with the incident beam:

$$
\beta_{h k l}=\frac{\pi}{2}-\arcsin \left(\frac{\lambda}{2 d_{h k l}}\right) .
$$

The ratio of the number of preferentially aligned planes to the corresponding number for a perfectly random sample can be expressed as $R$ in multiples of a random distribution (m.r.d.) with the function

$$
R=\frac{-\ln \left[I(\lambda) / I_{\text {base }}(\lambda)\right]}{z f_{h k l} \lambda^{2}},
$$

where $I(\lambda)$ is neutron transmission after traversing a sample of thickness $z, I_{\text {base }}$ is the base attenuation not affected by coherent scattering and $f_{h k l}$ is a materials parameter related to the structure factor (Kockelmann et al., 2007).

\subsection{Cross-correlation-based data analysis}

Sample anisotropy due to preferred orientation changes the shape of the Bragg edges (Santisteban, Edwards \& Stelmukh, 2006; Boin et al., 2011; Malamud et al., 2014). This presents a challenge to the analytical function in equation (3) in describing the shape and determining the position of the Bragg edge, since the function does not consider texture contributions in its parametrization. Rietveld-type analysis (Sato et al., 2011) and model-based fitting (Song et al., 2017) approaches have been attempted to solve this problem. In the absence of a validated analytical model for textured samples, a parameter-free cross-correlation-based analysis method has been developed and used here to estimate the shift of Bragg edges on both textured and non-textured samples. Crosscorrelation measures the similarity between two signals as a function of the shift of one relative to the other. The steps of performing the analysis are described below.

Firstly a reference Bragg edge and the Bragg edge from the region of interest, having approximately the same shapes (i.e. having similar preferred orientation), are selected [Fig. 3(a)]. Obtaining a reference Bragg edge with similar shape is achieved by measuring the unstressed part of the same sample, or by measuring a stress-relaxed coupon sectioned from the sample. Then, the reference and region-of-interest Bragg edges are selected from the same wavelength range, i.e. both have the same number of data points [Fig. 3(a)]. Secondly, since the edge-shaped transmission signal is not ideal for cross-correlation, the peak-shaped derivative of the signal is used. Numerical derivatives of both the reference Bragg edge and the Bragg edge from the region of interest are taken [Fig. 3(b)]. It has to be noted that differentiation amplifies noise. While the central difference method is used for the differentiation in the current work, there is a need for further studies including the use of higher-order differentiation methods with respect to the noise. Meanwhile, smoothing of the raw transmission data can be carefully carried out to help to mitigate the effects of noisy data; however, over-smoothing

Figure 3

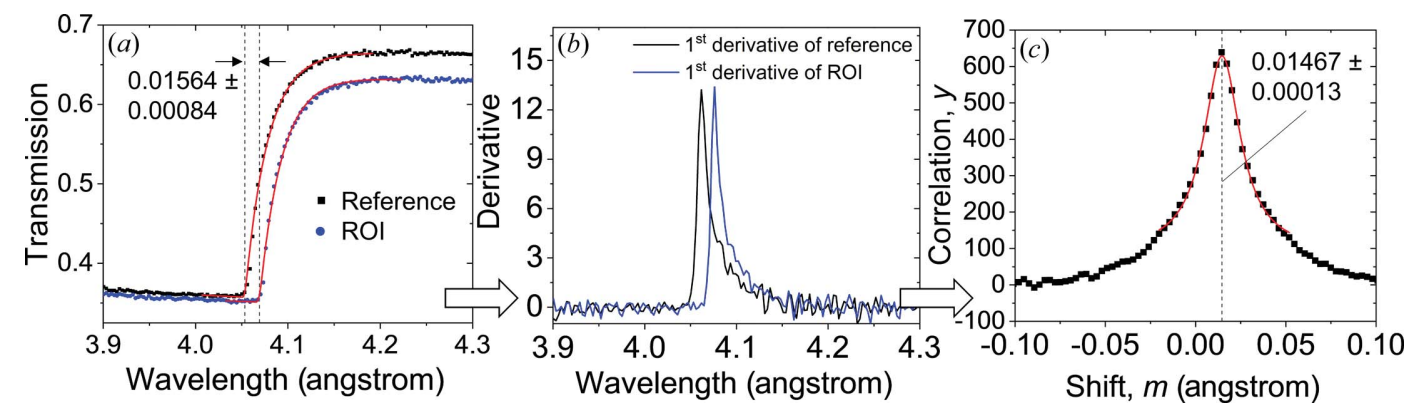

(a) Reference Bragg edge and Bragg edge from the region of interest (ROI), having a similar shape. The measured shift using the edge analytical function approach between the reference and the ROI Bragg edge is $0.01564 \pm 0.00084 \AA$. (b) First derivative of the reference and ROI Bragg edge. (c) Cross-correlation function of the reference and the ROI Bragg edge. The peak of the function represents the shift of the two Bragg edges, giving a value of $0.01467 \pm 0.00013 \AA$. 
(averaging over too many points) might add higher uncertainties to the final results of the cross-correlation.

The correlation coefficient $y$, as a function of the shift $m$ of the two Bragg-edge derivatives (reference and region of interest), is then calculated using equation (6):

$$
y(m)=\sum_{\lambda_{i}, i=0}^{M-1} f\left(\lambda_{i}\right) g\left(\lambda_{i-m}\right),
$$

where $f(\lambda)$ and $g(\lambda)$ are the two Bragg-edge derivatives and $M$ is the region of the wavelength range. The correlation of the derivatives in Fig. 3(b) is shown in Fig. 3(c). The position of the maximum of the correlation function $y(m)$ is assumed to provide the best estimate of the shift (i.e. difference in position) between the two Bragg edges. The correlation function can be obtained by peak fitting (in this case using a Voigt function) to determine the shift, while the fitting error can provide the shift uncertainty. Thus the shift between two Bragg edges is determined without requiring an analytical function. The cross-correlation analysis in Fig. 3(c) yields an edge shift of $0.01467 \pm 0.00013 \AA$, which agrees well with the result using the analytical function approach [Fig. 3(a)].

\section{Instrument characterization experiments}

A series of calibration experiments were performed to characterize the Bragg-edge imaging analysis on IMAT, in terms of accuracy and level of uncertainty of the result as well as the spatial resolution produced from the imaging. Different calibration powders were measured at room temperature to study the spectral resolution of the instrument. Measurements of a sample at different distances from the detector were performed to determine the achievable spatial resolution of the reconstructed Bragg-edge map. Transmission experiments were also performed at high temperature to investigate the accuracy and resolution of the Bragg-edge strain analysis performed on the instrument.

For all experiments, the flight path is approximately $56.37 \mathrm{~m}$, which is the distance from the moderator to the MCP detector. The neutrons were collected in histogram mode (neutron counts as a function of the neutron time of flight). The choppers were set to a frequency of $10 \mathrm{~Hz}$, providing a maximum wavelength of $7 \AA$, with the maximum intensity recorded at $\sim 2.6 \AA$ (Minniti et al., 2018). The wavelength band was divided into 2500-3000 bins, with a bin width of $\sim 0.0014 \AA$ (equivalent to $20 \mu \mathrm{s}$ ) for $\lambda<3.3 \AA$ and a bin width of $\sim 0.0029 \AA(40 \mu \mathrm{s})$ for $\lambda>3.3 \AA$. A narrower bin width at shorter wavelengths was applied in consideration of the sharper pulse widths.

\subsection{Calibration measurements}

To evaluate the instrumental effect on Bragg edges, calibration powder samples were used, assumed to have randomized crystallite orientation distributions and minimum internal strains. Four powder materials - cerium oxide $\left(\mathrm{CeO}_{2}\right)$, beryllium (Be), $\alpha$-iron $(\mathrm{Fe})$ and aluminium $(\mathrm{Al})$ powders were prepared. The $\mathrm{CeO}_{2}$ powder (Sigma Aldrich 211575
99.9\% trace metal basis, $<5 \mu \mathrm{m}$ powder) is commonly used as a standard to give a good approximation of instrument resolution since it has negligible intrinsic sample broadening. A sintered Be sample was selected since it can produce well separated and well defined Bragg edges in a relatively short measurement time. The Fe powder (Goodfellow FE006020 $99.0 \%$ purity, particle size $<60 \mu \mathrm{m}$ ) was used because of its high coherent cross section and its body-centred-cubic (b.c.c.) structure, producing well separated Bragg edges. The inclusion of $\mathrm{Al}$ powders (MBFiberglass IR350, 99.5\% purity) was to represent common engineering materials and a sample with weak coherent cross section. The $h k l$ lists and structure factors of the powders were obtained from nxsPlotter (Boin, 2012) and are included in Table 1. Each powder was held in a thinwalled $(1 \mathrm{~mm})$ aluminium container and placed $30 \mathrm{~mm}$ in front of the $\mathrm{MCP}$ detector. $\mathrm{CeO}_{2}, \mathrm{Fe}$ and $\mathrm{Al}$ powders were measured for about $5 \mathrm{~h}$, while the Be sample was measured for about $30 \mathrm{~min}$. A pinhole of $D=40 \mathrm{~mm}(L / D \simeq 250)$ was used for these measurements. In each case, the most intense Bragg edges were indexed (Table 1), and then the individual Bragg edges were fitted using equation (3).

The parameter $\tau$ from equation (3), related to the decay constant of the exponentially decaying 'storage' term of the moderator, was chosen as a sample-independent quantity to represent the instrument broadening. This is consistent with the descriptions of the wavelength-dependent resolutions of the diffraction-based ENGIN and ENGIN-X instruments at ISIS (Santisteban et al., 2001; Santisteban, Daymond et al., 2006) and with Monte Carlo simulations reported earlier for IMAT (Kockelmann et al., 2013; Burca et al., 2013), and an alternative choice to the often-quoted FWHM values of the peak shapes. The parameter $\tau$ is much larger, by up to a factor 5 , than the FWHM for a given wavelength, and as such better represents the instrument resolution of an imaging instrument. The parameter $\sigma$, instead, depends on both sample and instrumental setup. The resolution function of the IMAT instrument is reported in Section 4.1.

\subsection{Spatial resolution measurements}

Measurement of a 'Siemens star' in conjunction with a sample (Fig. 4) at different distances from the detector was performed. The purpose was to study the effect of both geometrical blurring and macro-pixel size on the spatial resolution of the reconstructed Bragg-edge map. The quarterSiemens star used was a thin neutron-absorbing pattern, made of $7 \mu \mathrm{m}$-thick gadolinium deposited on a silicon substrate (Pooley et al., 2017), with well defined spokes to measure spatial resolution. It was attached to the back of a $10 \times 10 \times$ $50 \mathrm{~mm} \mathrm{Fe}$ rod to provide sharp edges on a reconstructed Bragg-edge map. Using a pinhole of $D=80 \mathrm{~mm}(L / D=125)$, different sample-to-detector distances $l$ were applied to produce different geometrical blurring values $d$. Since the thickness of the foil in the transmission direction is negligible, it does not contribute to the $l$ value. The sample-to-detector distances were $l=25,75$ and $225 \mathrm{~mm}$, to produce $d$ values of 
Table 1

Lattice parameters $\lambda_{h k l}$ and structure factors $F$ of the calibration samples used in the experiment, with the most intense Bragg edges indexed.

The values are calculated using the nxs Plotter software (Boin, 2012). The product of $j^{*}\left(\lambda_{h k}^{2}\right)|F|^{2}$ represents the Bragg-edge intensity, with the values normalized to the most intense Bragg edge.

\begin{tabular}{|c|c|c|c|c|c|}
\hline Materials & Lattice parameter at room temperature $(\AA)$ & $\{h k l\}$ & $\lambda_{h k l}(\AA)$ & Multiplicity, $j$ & $j^{*}\left(\lambda_{h k}^{2}\right)|F|^{2}$ \\
\hline \multirow[t]{8}{*}{ Cerium oxide } & 5.411† (Kümmerle \& Heger, 1999; Harwood, 1949; Whitfield et al., 1966; Varez et al., 2007) & 111 & 6.250 & 8 & 191.3 \\
\hline & 5.412 (Wołcyrz \& Kepinski, 1992; Itoh et al., 2015) & 200 & 5.410 & 6 & 179.6 \\
\hline & 5.409 (Stecura \& Campbell, 1961) & 220 & 3.826 & 12 & 1000 \\
\hline & $5.4097 \pm 0.0007$ (current work) & 400 & 2.706 & 6 & 189.2 \\
\hline & & 422 & 2.210 & 24 & 382.9 \\
\hline & & 440 & 1.914 & 12 & 109.2 \\
\hline & & 620 & 1.712 & 24 & 133.3 \\
\hline & & 642 & 1.446 & 48 & 111.6 \\
\hline \multirow[t]{5}{*}{ Beryllium } & $a=2.286, c=3.584 \dagger($ Yang $\&$ Coppens, 1978) & 100 & 3.960 & 6 & 1000 \\
\hline & & 002 & 3.584 & 2 & 269.5 \\
\hline & & 102 & 2.658 & 12 & 838.8 \\
\hline & & 110 & 2.286 & 6 & 296.4 \\
\hline & & 112 & 1.928 & 12 & 392.5 \\
\hline \multirow[t]{8}{*}{ Iron } & $2.866 \dagger$ (Kochanovska, 1949; Jette \& Foote, 1935; Straumanis \& Kim, 1969) & 110 & 4.054 & 12 & 1000 \\
\hline & 2.867 (Owen \& Yates, 1933; Gorton et al., 1965) & 200 & 2.866 & 6 & 240.9 \\
\hline & 2.859 (Basinski et al., 1955) & 211 & 2.340 & 24 & 619.5 \\
\hline & $2.8675 \pm 0.0002$ (current work) & 220 & 2.026 & 12 & 223.9 \\
\hline & & 310 & 1.812 & 24 & 345.5 \\
\hline & & 321 & 1.532 & 48 & 459.3 \\
\hline & & 330 & 1.352 & 12 & 83.1 \\
\hline & & 431 & 1.124 & 48 & 198.6 \\
\hline \multirow[t]{6}{*}{ Aluminium } & $4.049 \dagger$ (Owen \& Yates, 1933; Swanson \& Tatge, 1953; Bourbia et al., 2010) & 111 & 4.676 & 8 & 1000 \\
\hline & 4.050 (Cooper, 1962; Figgins et al., 1956; Hull, 1917) & 200 & 4.050 & 6 & 550.6 \\
\hline & 4.039 (Wilson, 1941) & 220 & 2.864 & 12 & 504.6 \\
\hline & $4.0506 \pm 0.0008$ (current work) & 311 & 2.442 & 24 & 686.9 \\
\hline & & 222 & 2.338 & 8 & 205.4 \\
\hline & & 331 & 1.858 & 24 & 333.8 \\
\hline
\end{tabular}

$\dagger$ Used for input parameter in the nxsPlotter software.

$0.2,0.6$ and $1.8 \mathrm{~mm}$, respectively, according to equation (1). Each measurement was performed for $2 \mathrm{~h}$.

In the first analysis, white-beam radiographs (averaged over the whole wavelength range) were taken with different $d$ values. From the radiographs, a line profile of neutron intensity was drawn on the edge of the Siemens star, and a modulation transfer function (MTF) was derived (Nikolay Kardjilov, personal communication, 1 June 2016). A $10 \%$ contrast cut-off was applied to define the highest achievable

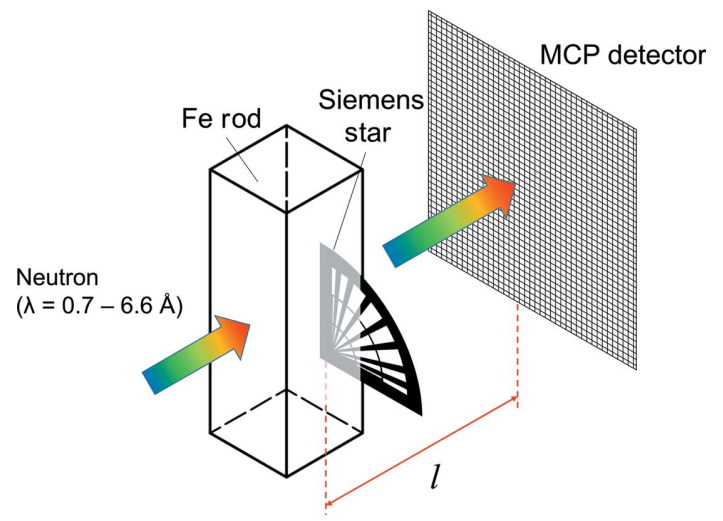

Figure 4

Experimental setup of spatial resolution measurement. The distances $l$ between the Siemens star (attached to the back of the Fe rod) and the detector were 25,75 and $225 \mathrm{~mm}$. spatial frequency, and the inverse of this yields the maximum achievable spatial resolution. The method has been used to determine spatial resolutions in neutron imaging (Treimer, 2009). This analysis determined the spatial resolution due to geometrical blurring. In the second analysis step, Bragg-edge maps were reconstructed from the data using TPX_EdgeFit with different macro-pixel sizes ranging from $5 \times 5$ to $50 \times 50$ pixels. The Bragg-edge height [coefficient $C_{2}$ of Tremsin et al. (2009)] map was subjected to an MTF analysis using the edge of the Siemens star covering the Fe rod sample. The spatial resolution of the macro-pixel method was determined from the analysis and is reported in Section 4.2.

\subsection{High-temperature measurement}

A neutron transmission experiment at elevated temperatures was performed on IMAT. The thermal expansions of several samples were measured to provide data from stressfree strains in the materials, which could be used to determine the level of uncertainty and the accuracy of the overall measurement system by comparing the measured values against well documented thermal lattice strain data. $\mathrm{CeO}_{2}, \mathrm{Fe}$ and pure $\mathrm{Al}$ powders were used in the high-temperature experiment, representing measurement of ceramics and nontextured engineering metals. A rolled Al1050 plate (BS EN 
573:3 1050A H14) with a nominal thickness of $6 \mathrm{~mm}$ was also measured to represent textured engineering alloys.

The $\mathrm{CeO}_{2}, \mathrm{Al}$ and $\mathrm{Fe}$ powder samples were inserted into $15 \mathrm{~mm}$-diameter vanadium cans of $0.15 \mathrm{~mm}$ wall thickness, while the textured $\mathrm{Al}$ plate was cut into a disc with $30 \mathrm{~mm}$ diameter. These samples were then loaded into a RAL5 furnace available at the ISIS facility, which was installed in front of the MCP transmission detector. The RAL5 furnace allows neutron transmission measurement at temperatures up to $1273 \mathrm{~K}$ by means of radiative heating with a vanadium foil element heater. A vacuum better than $10^{-3}$ mbar $(0.1 \mathrm{~Pa})$ was applied to avoid oxidation of the samples, while the vanadium element and vanadium windows in heat shields and furnacevessel walls allowed neutrons to pass through with minimal Bragg diffraction. Two thermocouples, one at the bottom of the heating element and another attached to the sample, were fed into a proportional-integral-derivative (PID) temperature controller to record and regulate the sample temperature. Owing to the installation of the furnace on the beamline, the distance between the sample and the MCP detector was $170 \mathrm{~mm}$. Measurements were taken in situ at a range of temperatures from room temperature up to $1143 \mathrm{~K}$ for $\mathrm{Fe}$ and $\mathrm{CeO}_{2}$, and up to $823 \mathrm{~K}$ for the $\mathrm{Al}$ samples. The temperature was held constant during each measurement within $2 \mathrm{~K}$, with a measurement time of $3 \times 0.5 \mathrm{~h}(1.5 \mathrm{~h}$ in total $)$ for the $\mathrm{Fe}$ and $\mathrm{CeO}_{2}$ samples and $4 \times 0.5 \mathrm{~h}(2 \mathrm{~h}$ in total $)$ for the $\mathrm{Al}$ sample. The $0.5 \mathrm{~h}$ data from each measurement were analysed individually and the results showed good repeatability. The sum of the $0.5 \mathrm{~h}$ data from each measurement was taken to improve the statistics and was used for the analysis in this paper. The experimental setup of the high-temperature measurements is illustrated in Fig. 5

The uncertainty of the measured strain corresponds to the uncertainty in the determination of the Bragg-edge position: this is similar to Bragg-peak analysis for strain measurement (Santisteban, Daymond et al., 2006). The strain was determined using equation (7) and the strain uncertainty was calculated following the error propagation expressed in equation (8):

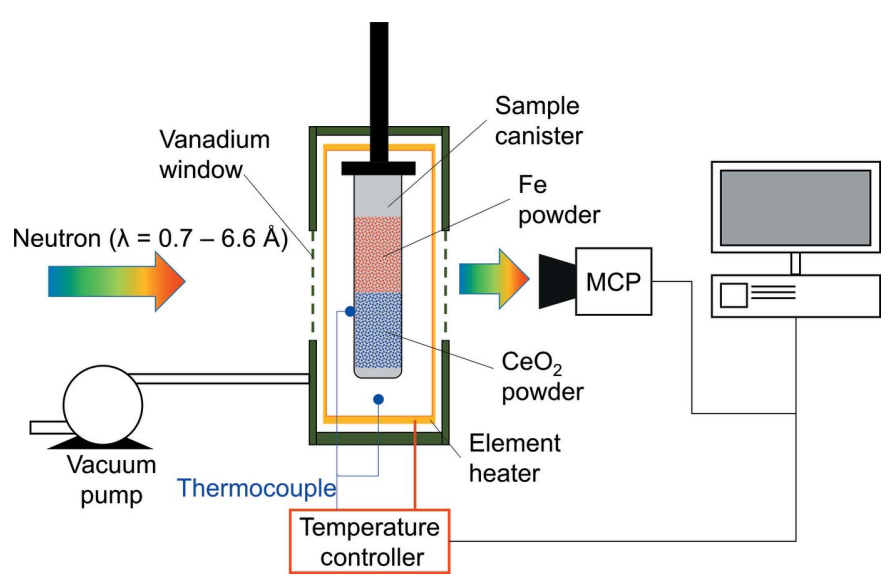

Figure 5

Setup of high-temperature experiment on IMAT, with simultaneous measurement of $\mathrm{CeO}_{2}$ and $\mathrm{Fe}$ powders.

$$
\begin{gathered}
\varepsilon=\frac{\lambda_{T}-\lambda_{0}}{\lambda_{0}}, \\
\Delta \varepsilon=\left[\frac{1}{\lambda_{0}^{2}}\left(\Delta \lambda_{T}\right)^{2}+\frac{\lambda_{T}^{2}}{\lambda_{0}^{4}}\left(\Delta \lambda_{0}\right)^{2}\right]^{1 / 2},
\end{gathered}
$$

where $\lambda_{0}$ and $\lambda_{T}$ are the fitted parameters of equation (3) describing the position of a Bragg edge at room temperature and at a particular temperature $T$, respectively; and $\Delta \lambda_{0}$ and $\Delta \lambda_{T}$ are the fitting uncertainties. Any $T$ values can be used for uncertainty determination; in this case $T=573 \mathrm{~K}$. The Bragg edge being analysed was Fe $\{110\}$. The uncertainty of measurement as a function of time and macro-pixel size was analysed. To assess the accuracy of the strain measurement, the thermal lattice expansions of the $\mathrm{Fe}, \mathrm{CeO}_{2}$ and $\mathrm{Al}$ powders measured using neutron transmission were compared against literature data (Basinski et al., 1955; Stecura \& Campbell, 1961; Wilson, 1941; Jette \& Foote, 1935; Davey, 1925). In the current work, lattice parameters were determined by fitting equation (3) to $\mathrm{Fe}\{110\}, \mathrm{CeO}_{2}\{220\}$ and $\mathrm{Al}\{111\}$ Bragg edges. The uncertainty and the accuracy of strain measurement on IMAT is reported in Section 4.3.

To assess the accuracy of the cross-correlation method in determining changes in lattice spacing, the method was used to measure the thermal lattice strain $\varepsilon_{T}$ on both textured and non-textured samples:

$$
\varepsilon_{T}=\frac{\Delta a}{a_{0}}=\frac{a_{T}-a_{0}}{a_{0}},
$$

where $a_{T}$ is the lattice parameter at temperature $T$ and $a_{0}$ is the lattice parameter at room temperature. Bragg-edge shift values obtained from the cross-correlation analysis are represented by $\Delta a$. Via cross-correlation, thermal strain was measured from texture-free powder samples using Fe $\{110\}$ and $\mathrm{CeO}_{2}\{220\}$ Bragg edges and from the textured aluminium plate using the $\mathrm{Al}\{111\}$ Bragg edge. The results are compared against thermal strain analysed using nonlinear fitting methods. The accuracy of the cross-correlation method is presented in Section 4.4 .

\section{Results}

\subsection{IMAT resolution function}

The aim of this section is to report the experimental evaluation of the IMAT wavelength resolution and provide calibration values as a guide for Bragg-edge analysis on the instrument. Fig. 6(a) presents the pulse width of IMAT as a function of neutron wavelength. The data points are the decay constant parameters $\tau$ from equation (3) for the different Bragg edges of the calibration powders, with $\{h k l\}$ values as indexed in Table 1. The size of the detection area, i.e. the number of pixels from which the Bragg edges were extracted, was $478 \times 478$ for $\mathrm{CeO}_{2}, 346 \times 346$ for $\mathrm{Be}, 337 \times 337$ for $\mathrm{Al}$ and $274 \times 274$ for Fe. A sigmoidal logistic function [described in equation (10), where $\tau$ is in $\mu$ s and $\lambda$ is in $\AA$ ] was used to simultaneously fit the $\mathrm{Fe}$ and $\mathrm{CeO}_{2}$ data points (the ones with the lowest uncertainties) as shown by the red curve in Fig. 6(a). 


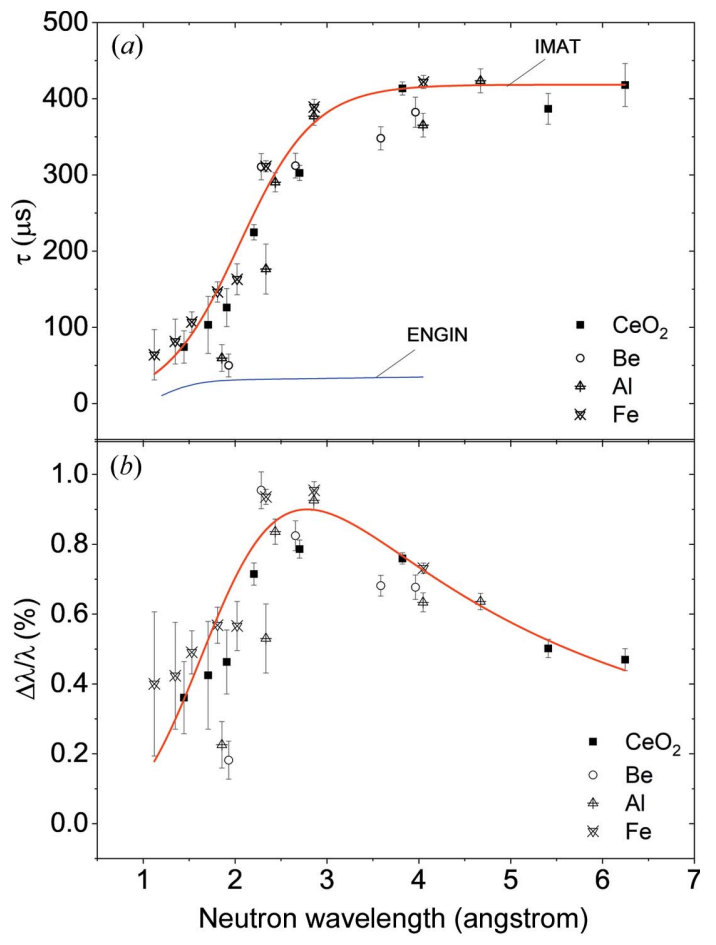

Figure 6

(a) IMAT decay constant $\tau$ as a function of neutron wavelength, determined for different calibration powders. The red curve is the sigmoidal fit described by equation (10). Comparison with ENGIN (Santisteban et al., 2001) emphasizes the characteristics of the 'broadpulse' coupled moderator for IMAT compared with the 'sharp-pulse' decoupled moderator for ENGIN-X. (b) Resolution function of the IMAT beamline.

Fig. 6(b) presents the resolution function $(\Delta \lambda / \lambda)$ of the instrument, which is obtained by dividing the parameter $\tau$ by the corresponding TOF position for each Bragg edge. The red curve shows the sigmoidal fit to the $\mathrm{Fe}$ and $\mathrm{CeO}_{2}$ data points. The fit shows that the worst resolution of $\sim 0.9 \%$ is observed at a wavelength of $\sim 2.6 \AA$.

$$
\tau=\frac{418}{[1+155.1 \exp (-2.46 \lambda)]} .
$$

The determination of wavelength resolution carried out in this work with different calibration powders agrees well with previous measurements on IMAT (Minniti et al., 2018). Moreover, the $\tau$ curve in Fig. 6(a) can be used as a sampleindependent constraint when performing Bragg-edge fitting. Meanwhile, the experimental results in Fig. 6(b) also agree fairly well with and validate the modelling of the IMAT beamline using Monte Carlo simulations (Kockelmann et al., 2013; Burca et al., 2013).

Fig. 6(a) also demonstrates the effect of a different moderator on the wavelength resolution. Using a coupled liquid-hydrogen moderator, IMAT has greater values by almost a factor of ten compared with ENGIN which uses a decoupled liquid-methane moderator. The same decoupled moderator is used by ENGIN-X. Consequently, IMAT produces broader Bragg edges than ENGIN-X, as can be observed from Fig. 2. On the other hand, the use of the coupled moderator allows IMAT to have a much higher flux than ENGIN-X, supporting IMAT's main purpose as an imaging beamline.

\subsection{Spatial resolution of Bragg-edge map}

Fig. 7(a) shows an example of a radiograph of the Siemens star behind an Fe rod with geometrical blurring of $d=0.2 \mathrm{~mm}$ with the corresponding MTF. A $10 \%$ contrast cut-off, as indicated in Fig. 7(b), gives a spatial frequency of 5.6 line pairs (lp) per millimetre, which corresponds to a spatial resolution of $89 \mu \mathrm{m}$. Analysing the MTF for the radiographies with two other $d$ values of 0.6 and $1.8 \mathrm{~mm}$ yields spatial resolution values of 240 and $767 \mu \mathrm{m}$, respectively. This spatial resolution variation is exclusively due to the geometrical blurring (i.e. due to the change of $l, L$ or $D$ ) and represents the spatial resolution of maps for which the macro-pixel method is not applied (e.g. in texture mapping analysis in Section 5.2). As expected, larger nominal values of geometrical blurring $d$ (in this case due to the increase of sample-to-detector distance, $l$ ) produced lower (higher nominal) spatial resolution.

The effective spatial resolution of the reconstructed Braggedge map is influenced by both the geometrical blurring and the macro-pixel size. To determine this effective spatial
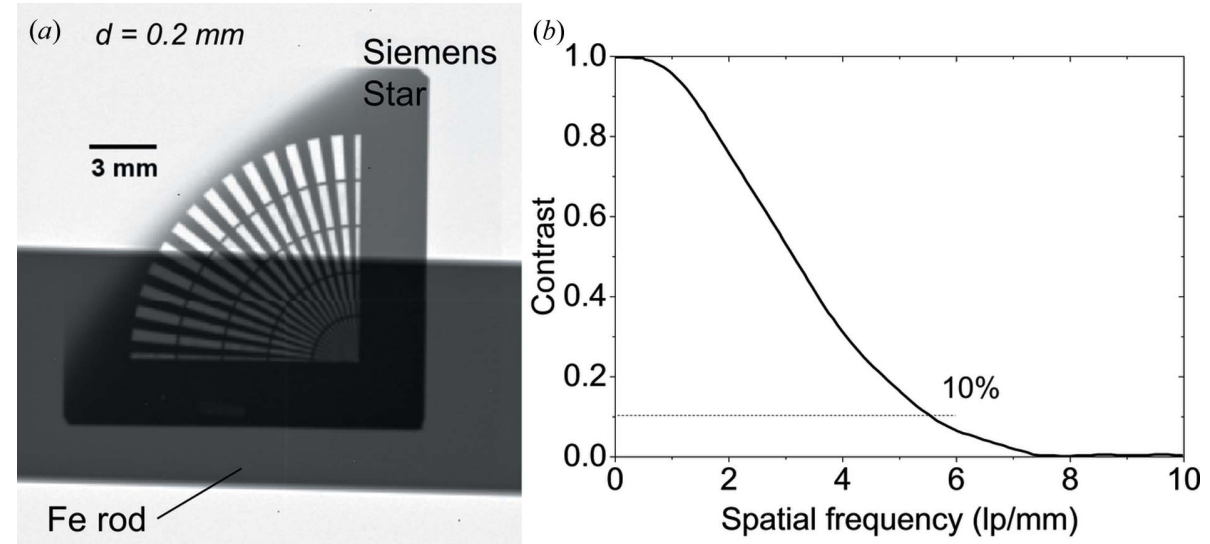

Figure 7

(a) Radiography of the Siemens star behind the $10 \mathrm{~mm}$ Fe rod measured with a sample-to-detector distance of $25 \mathrm{~mm}(d=0.2 \mathrm{~mm})$. $(b)$ Corresponding MTF with a contrast cut-off of $10 \%$, producing a spatial frequency of 5.6 line pairs per millimetre, equivalent to a spatial resolution of $89 \mu \mathrm{m}$. 


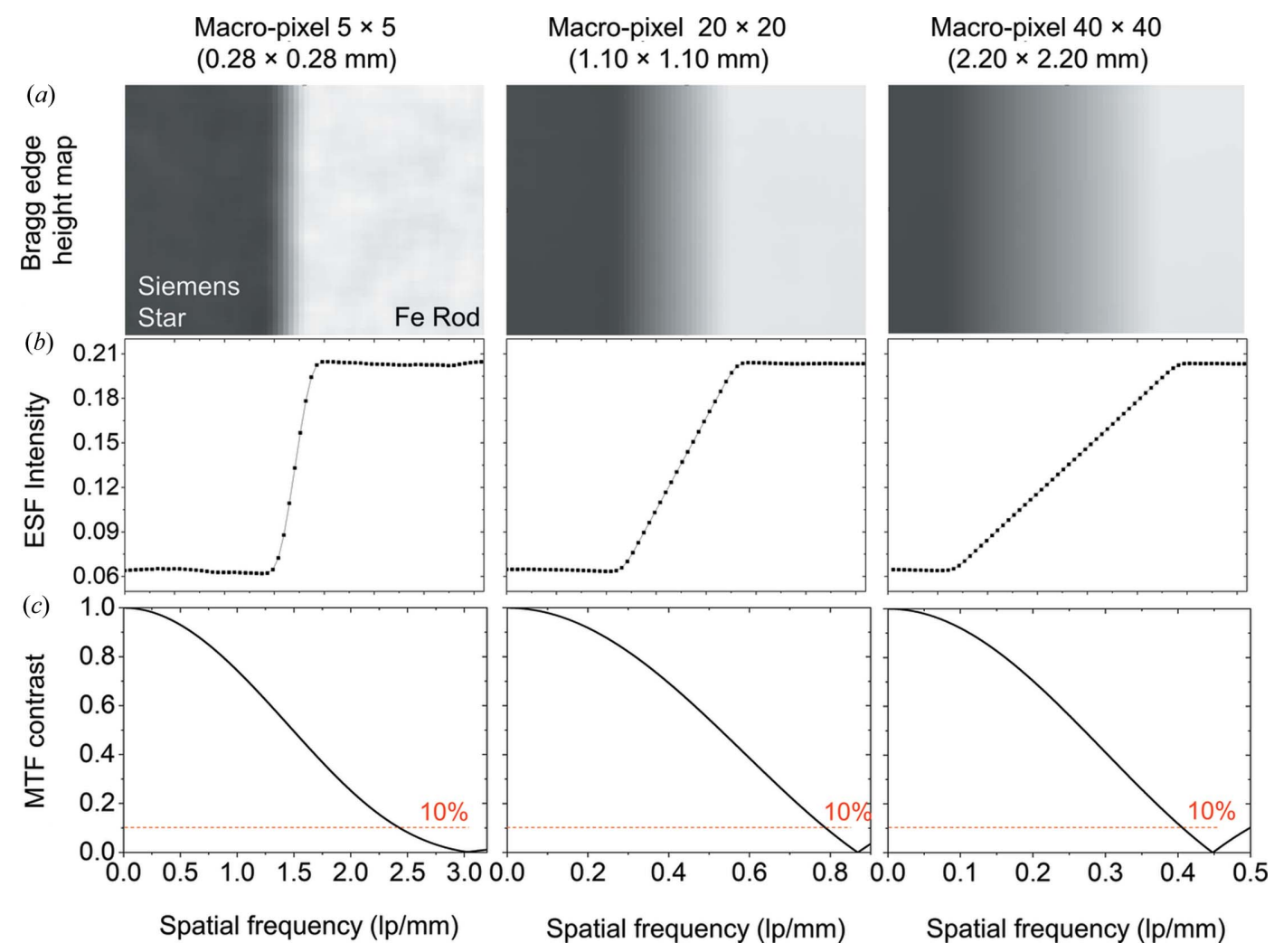

Figure 8

Spatial frequency $(\mathrm{Ip} / \mathrm{mm})$

Spatial frequency $(\mathrm{Ip} / \mathrm{mm})$

(a) Bragg-edge height maps of the Siemens star edge behind the Fe rod, reconstructed from the $\{110\}$ edge using macro-pixels of $5 \times 5,20 \times 20$ and $40 \times$ 40, with corresponding ESF $(b)$ and MTF $(c)$. The reconstruction was performed using data measured with $d=0.2 \mathrm{~mm}$.

resolution, the MTFs with $10 \%$ contrast cut-off were calculated for (i) Bragg-edge height maps reconstructed using different macro-pixel sizes and (ii) data with different geometrical blurring. Fig. 8(a) presents examples of Braggedge height maps reconstructed using $5 \times 5,20 \times 20$ and $40 \times$ 40 macro-pixels for data with $d=0.2 \mathrm{~mm}$, showing the Siemens star edge over the map of the $\{110\}$ Bragg edge of the Fe rod. The corresponding edge spread function (ESF) and MTF (Cao $\&$ Biegalski, 2007) are shown in Figs. 8(b) and 8(c), respectively, where a $10 \%$ contrast cut-off is again used as the criterion for spatial resolution.

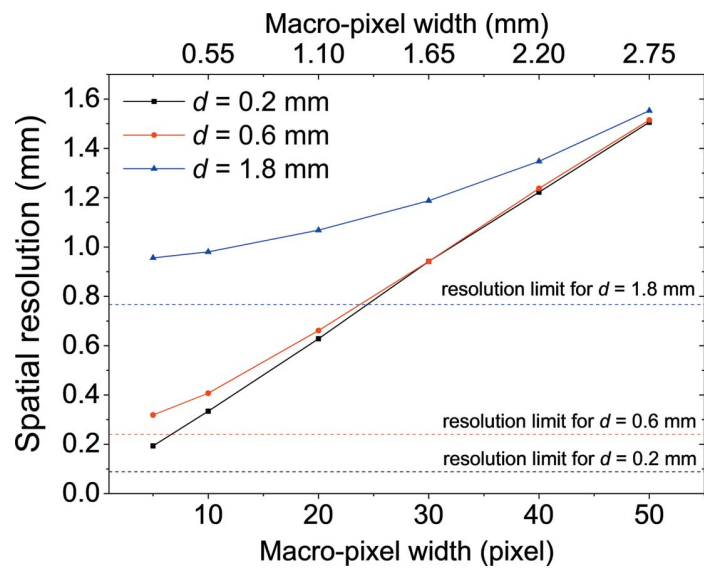

Figure 9

Spatial resolution of a Bragg-edge map as a function of macro-pixel size, reconstructed from data with different geometrical blurring values $d$.
The results are shown in Fig. 9. It can be observed that for $d=0.2 \mathrm{~mm}$ the relation between effective spatial resolution and macro-pixel size is almost linear. For $d=0.6 \mathrm{~mm}$, however, the improvement of spatial resolution due to the reduction of macro-pixel size become less significant, especially with the application of macro-pixels smaller than $30 \times 30$ pixels (Fig. 9). This is due to the geometrical blurring $d$ limiting the maximum achievable spatial resolution, shown by the dashed line in Fig. 9. As indicated in Fig. 9, the trend is further observed for $d=1.8 \mathrm{~mm}$, where reduction of the macro-pixel width by a factor of 10 (i.e. from 50 to 5 pixels) only improved the spatial resolution by around $40 \%$ (i.e. from 1.55 to $0.96 \mathrm{~mm}$ ). The results show that the use of smaller macro-pixel sizes is desirable up to a point where geometrical blurring limits the achievable spatial resolution, and this fact therefore needs to be considered when designing experiments. Fig. 9 also implies that the spatial resolution is better than the macro-pixel size, as a result of using a running average with $55 \mu \mathrm{m}$ (1 MCP pixel) shift between each reconstructed point (Tremsin et al., 2018). This is equivalent to an ideal $55 \mu \mathrm{m}$ spatial resolution strain map blurred by a running Gaussian filter (Tremsin, Ganguly et al., 2016), and therefore the map has higher spatial resolution than the original macro-pixel size.

\subsection{Uncertainty and accuracy of Bragg-edge strain measurement}

Analysis of the uncertainty and accuracy of Bragg-edge strain mapping on IMAT was performed using the data 
obtained from high-temperature measurements (Section 3.3). Fig. 10 shows examples of neutron transmission spectra obtained from a non-textured sample (in this case Fe powder) and a textured sample (rolled Al plate) at different temperatures. Fig. 10(a) shows multiple Bragg edges from different sets of $\mathrm{Fe}\{h k l\}$ which shift to higher wavelengths with the increase of temperature, as a result of thermal expansion of the material. Moreover, the Bragg-edge heights (the difference in transmitted intensity above and below the edge) decrease as the temperature increases. This observation relates to the thermal motion of atoms and will be further discussed in Section 5.3. It can also be observed that, beyond the Bragg cut-off at $4.05 \AA$, i.e. the wavelength above the $\{110\}$ Bragg edge for $\mathrm{Fe}$, the transmission decreases as the temperature increases owing to the increase in thermal diffuse scattering (TDS), while absorption does not change with temperature significantly apart from a change in density. As TDS increases and Bragg diffraction decreases with rising temperature, the change in transmission is less pronounced below the Bragg cut-off. Fig. 10(b) shows transmission spectra through the whole thickness of the rolled Al plate. Spectra measured from room temperature to $573 \mathrm{~K}$ show irregular Bragg-edge shapes, implying the presence of crystallographic texture. This is especially apparent for the $\{111\}$ Bragg edge as shown in the inset of Fig. 10(b). At $623 \mathrm{~K}$, however, the transmission spectrum changes drastically, more closely resembling data from a randomly oriented sample. A spectrum from a completely non-textured sample, i.e. Al powder, is plotted for comparison. This change is due to recrystallization at higher temperature and will be discussed further in Section 5.2.

To investigate the uncertainty and the strain resolution, $\mathrm{Fe}$ $\{110\}$ Bragg edges were analysed. The strain-affected Bragg edges were acquired from $\mathrm{Fe}$ powder data measured at $T=$ $575 \mathrm{~K}$ for $1.5 \mathrm{~h}$ (actual measurement time, $t_{\text {act }}$ ). Bragg edges were obtained from different sets of $n \times n$ pixels (actual area,
$\left.A_{\text {act }}\right)$ ranging from $4 \times 4$ to $220 \times 220$ pixels to obtain the uncertainty as a function of statistical quality. The reference Bragg edge was taken from $\mathrm{Fe}$ powder data measured at room temperature. When acquiring the reference Bragg edge, a large detection area size was selected in order to obtain good statistics. This reflects the actual experimental conditions, where the reference or $d_{0}$ sample is usually measured for a longer time to achieve the best statistics possible to minimize the strain uncertainty. The positions of the reference and the strain-affected Bragg edges, $\lambda_{0}$ and $\lambda_{T}$, respectively, were determined by the analytical function described by equation (3). The fitting uncertainties $\Delta \lambda_{0}$ and $\Delta \lambda_{T}$ were then used to calculate the strain uncertainty using equation (8). For experimental designs, the interest is in the determination of the minimum achievable uncertainty and the time needed to achieve different levels of uncertainty. Therefore, the uncertainty as a function of statistical quality is represented in terms of macro-pixel size and effective measurement time $t_{\text {eff }}$. The four macro-pixel sizes of interest are $10 \times 10,20 \times 20,50 \times 50$ and $100 \times 100$, while $t_{\text {eff }}$ is calculated using equation (11):

$$
t_{\text {eff }}=\frac{A_{\text {act }} t_{\text {act }}}{\text { macro }- \text { pixel size }} \text {. }
$$

The results are shown in Fig. 11. The curves flatten around the value of $80-90 \mu \varepsilon$, indicating the strain resolution. Using a macro-pixel of $20 \times 20$, an uncertainty of $125 \mu \varepsilon$ can be achieved within $10 \mathrm{~h}$ and an uncertainty of $200 \mu \varepsilon$ is achievable within $3 \mathrm{~h}$ of measurement time. Note, however, that these numbers are valid only for the given measurement conditions, i.e. specific to strain analysed from the most intense Fe $\{110\}$ Bragg edges with a sample thickness of $15 \mathrm{~mm}$ and $L /$ $D$ of 250 . The latter gives an average neutron count rate of 1 neutron per second per $40 \mu$ s TOF bin for a $20 \times 20$ macropixel, with a detector efficiency of $40 \%$. Different uncertainty plots, as displayed in Fig. 11, are expected for different Bragg edges and/or different sample geometries. An $L / D$ of 250

Figure 10
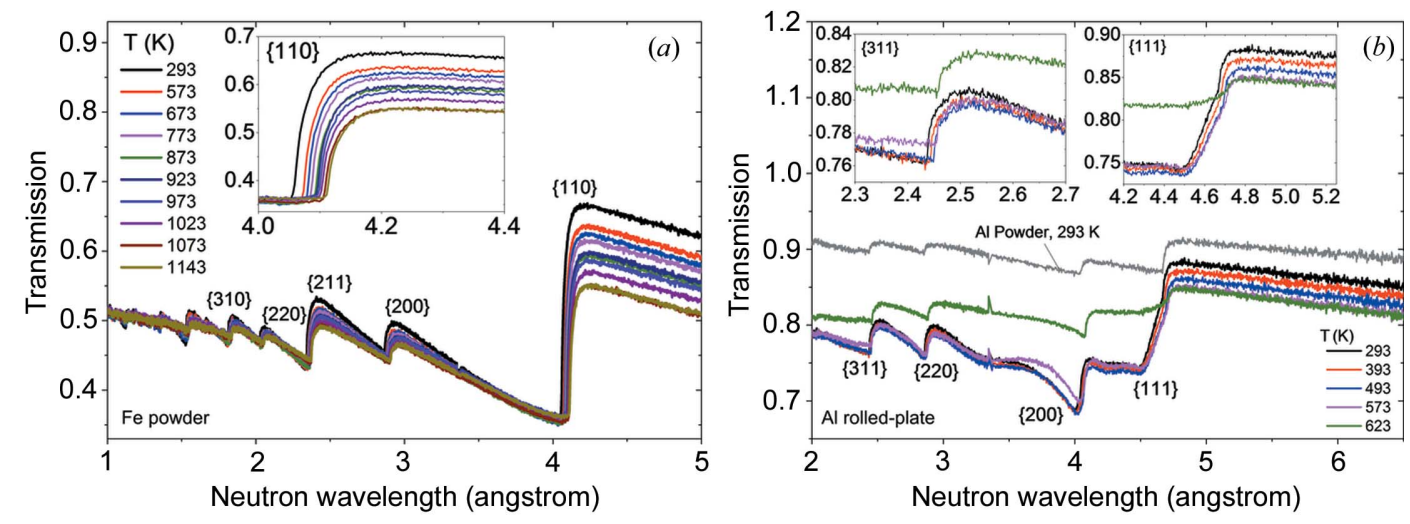

(a) Transmission spectra of Fe powder at different temperatures. The Bragg edges shift to higher wavelengths with increasing temperature because of thermal lattice expansion. The Bragg-edge heights decrease with the increase of temperature because of the thermal motion of atoms. The typical detection-area size for the spectra was $\sim 256 \times 256$ pixels. $(b)$ Bragg-edge spectra taken from the whole thickness section of the rolled aluminium plate (detection-area size of $\sim 200 \times 200$ pixels, sample thickness in transmission direction of $30 \mathrm{~mm}$ ). From room temperature to $573 \mathrm{~K}$, the spectra exhibit irregular Bragg-edge shapes due to strong crystallographic texture in the sample. At $623 \mathrm{~K}$, the transmission spectra change drastically, indicating recrystallization. Spectra from a non-textured Al powder are plotted for comparison, with higher transmission due to smaller sample thickness (detection area size of $\sim 268 \times 268$ pixels, sample thickness in transmission direction of $15 \mathrm{~mm}$ ). 
provides a good compromise between spatial resolution and collection time for many IMAT experiments. The use of larger pinhole sizes (i.e. 80 or $100 \mathrm{~mm}$ ) has already significantly reduced the measurement times on IMAT (by a factor of four or more) but with the penalty of lower spatial resolution due to geometrical blurring, as mentioned in Section 4.2. Ultimately, the strain uncertainty can be improved by implementing a multiple-Bragg-edge refinement, which is the intention for the future.

Lattice parameters obtained for $\mathrm{Fe}, \mathrm{CeO}_{2}$ and $\mathrm{Al}$ powders at room temperature were compared against the literature values. Using neutron transmission, the lattice parameter of $\mathrm{Fe}$ was measured to be $2.8675 \pm 0.0002 \AA$. This value agrees well with literature values listed in Table 1 . The lattice parameters of $\mathrm{CeO}_{2}$ and $\mathrm{Al}$ measured by neutron transmission are 5.4097 $\pm 0.0007 \AA$ and $4.0506 \pm 0.0008 \AA$, respectively. These values also agree well with literature values in Table 1 . The agreement between the current work and literature values demonstrates the accuracy of neutron transmission for latticeparameter determination.

In order to give a measure of the accuracy of a strain measurement using neutron transmission, the lattice-parameter values of the $\mathrm{Fe}, \mathrm{CeO}_{2}$ and $\mathrm{Al}$ powders at different temperatures from the current work are plotted against literature values (Fig. 12). Despite showing differences in the absolute lattice-parameter values, particularly for $\mathrm{Fe}$ and $\mathrm{Al}$, the present results agree very well with the literature for the relative changes of lattice parameter as a function of temperature. This is shown by fitting a third-order polynomial to describe thermal expansion, to both the current measurements and literature values. The fitted coefficients are given in Fig. 12. For $\mathrm{Fe}$ and $\mathrm{Al}$ data, the expansion coefficients compare well with literature values, most of them within the fitting error [Figs. 12(a) and 12(c), respectively]. For the $\mathrm{CeO}_{2}$ data, good agreement can be observed between the present results and literature values for all the fit parameters. Discrepancies in intercepts, i.e. absolute lattice parameters at room temperature, with results from Basinski et al. (1955) and Wilson (1941) [Figs. 12(a) and 12(c)] are conceivably due to the different amounts of impurities in the samples used. Note

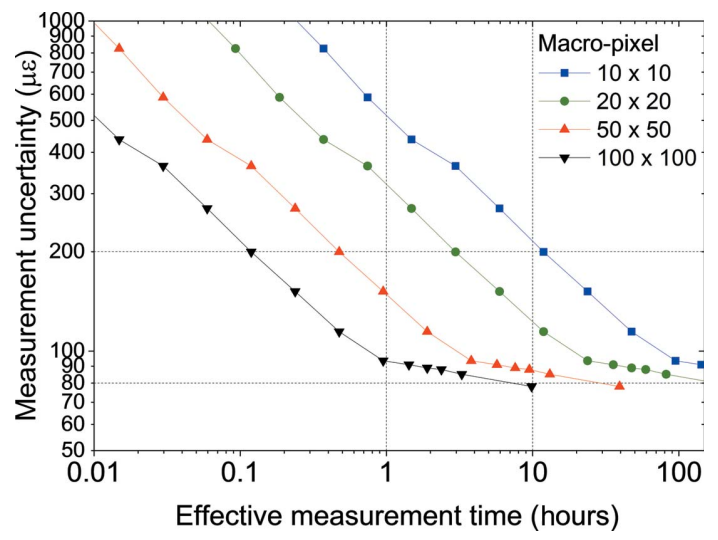

Figure 11

Strain resolution analysed from the Fe $\{110\}$ Bragg edge as a function of effective measurement time. that the two aforementioned literature comparisons were selected since both provide thermal expansions in a temperature range comparable to the current work. Meanwhile, the good agreement between relative changes of lattice parameter as a function of temperature measured in the current work and in previous literature for all three samples (Fig. 12) is a confirmation of the good accuracy and quantifies the strain uncertainties for the neutron transmission measurements on IMAT.
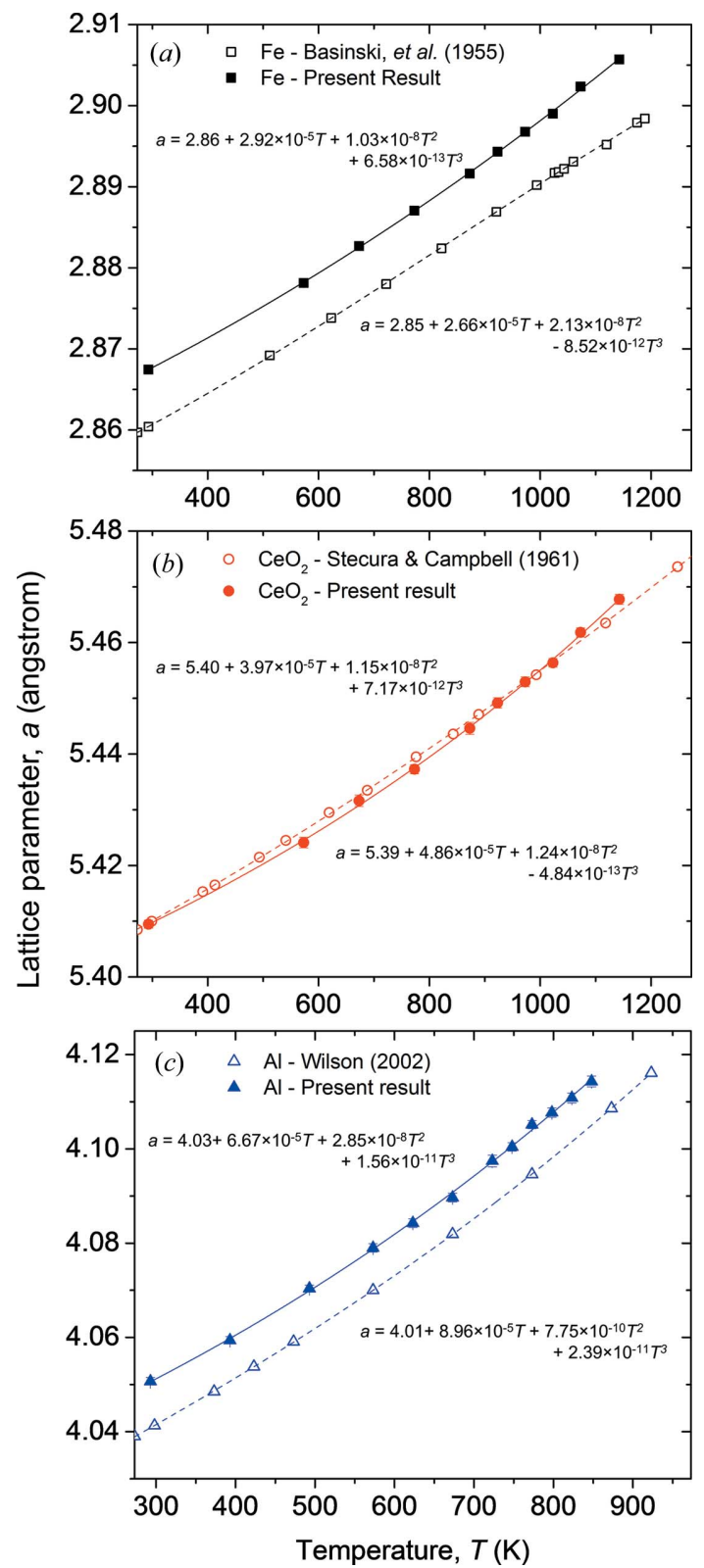

Figure 12

Lattice parameters of $(a) \mathrm{Fe},(b) \mathrm{CeO}_{2}$ and $(c) \mathrm{Al}$ powders as a function of temperature, measured in the current study by neutron transmission at IMAT (solid symbols), and compared with literature values (open symbols). Solid and dotted lines are third-order polynomial fits with the equation given on the graphs. The fitted Bragg edges were extracted from detection-area sizes of $256 \times 256$ pixels for $\mathrm{Fe}, 230 \times 230$ pixels for $\mathrm{CeO}_{2}$ and $268 \times 268$ pixels for $\mathrm{Al}$. 


\subsection{Cross-correlation for Bragg-edge shift estimation}

Fig. 13 shows thermal lattice strains of $\mathrm{Fe}$ and $\mathrm{CeO}_{2}$ powders analysed by Bragg-edge fitting and the crosscorrelation method. The results lie on a straight line with a slope of $0.983 \pm 0.005$ and $0.952 \pm 0.015$ for $\mathrm{Fe}$ and $\mathrm{CeO}_{2}$ data, respectively, indicating that the cross-correlation method and the analytical Bragg-edge fitting method yield consistent results for the non-textured sample. It can be observed as well that the level of uncertainty that is achieved by crosscorrelation is similar to that by Bragg-edge fitting.

While it was shown that cross-correlation analysis works for non-textured samples, the main purpose was to demonstrate that this method works for measuring shifts of Bragg edges with irregular shapes due to the presence of crystallographic texture. The analysis was performed for two Bragg edges of the same transmission spectrum taken from the textured Al1050 plate: $\mathrm{Al}\{111\}$ and $\mathrm{Al}\{311\}$. From the spectra shown in the inset of Fig. 10(b), it can be seen that the $\mathrm{Al}\{311\}$ edge is a more regular 'powder-like' Bragg edge compared with the Al $\{111\}$ edge for which an almost linear increase of transmission is observed ahead of the Bragg edge itself. The shift of the two

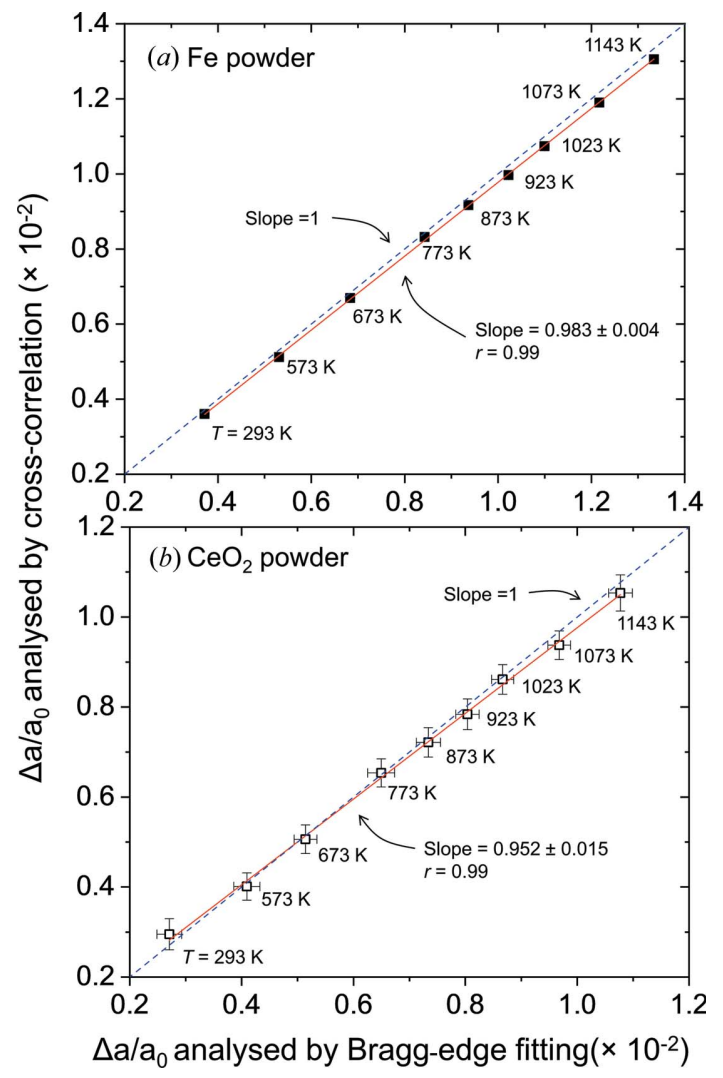

Figure 13

Thermal lattice strains $\Delta a / a_{0}$ of $(a) \mathrm{Fe}$ and $(b) \mathrm{CeO}_{2}$, calculated using equation (9), analysed using cross-correlation and Bragg-edge fitting methods. The red lines are the linear fit of the data points, while the blue dashed lines are lines with a slope of 1 , shown for comparison. The results indicate that cross-correlation successfully reproduces the Bragg-edge fitting results for a non-textured sample. The Bragg-edge spectra for both approaches were extracted from detection-area sizes of $256 \times 256$ pixels for $\mathrm{Fe}$ and $230 \times 230$ pixels for $\mathrm{CeO}_{2}$. edges due to thermal expansion is the same for the cubic unit cell of aluminium. Therefore, the more established edgefunction fit approach was applied to measure the shift of the $\mathrm{Al}\{311\}$ edges, and the results were used as the reference to benchmark the cross-correlation method for analysing the shift of $\mathrm{Al}\{111\}$ edges.

Fig. 14 shows that cross-correlation analysis on the irregularly shaped Al $\{111\}$ Bragg edge produces thermal lattice strains that agree well with the reference, the differences being well within the uncertainty of the measurement. Meanwhile, thermal lattice strains from the Bragg-edge fitting method on the textured $\{111\}$ deviate significantly from the reference, although this is partly captured in the increased measurement uncertainty. This implies that the cross-correlation analysis better reproduces the strain measurement on a textured sample and may be used in cases where the more conventional analytical Bragg-edge function-fitting approach is hampered by irregularly shaped Bragg edges.

In the presence of texture gradients, e.g. across the thickness of a rolled aluminium plate, thickness-specific reference Bragg edges have to be used to match the shape of the Bragg edge in the region of interest. Good statistical precision of the reference Bragg edge is desirable to minimize the uncertainty of the final result, in the same way as is needed for the conventional Bragg-edge fitting method. While smoothing of the transmission signals prior to taking derivatives can help overcome the noise introduced by the differentiation, the current application of the technique is mainly for analysis of data with good statistical quality, which usually means averaging is required over a relatively large area of the sample. With the ongoing development of neutron instrumentation and detector technology, however, the statistical significance of neutron data is likely to improve, with scope to implement cross-correlation for strain mapping. More work is needed to

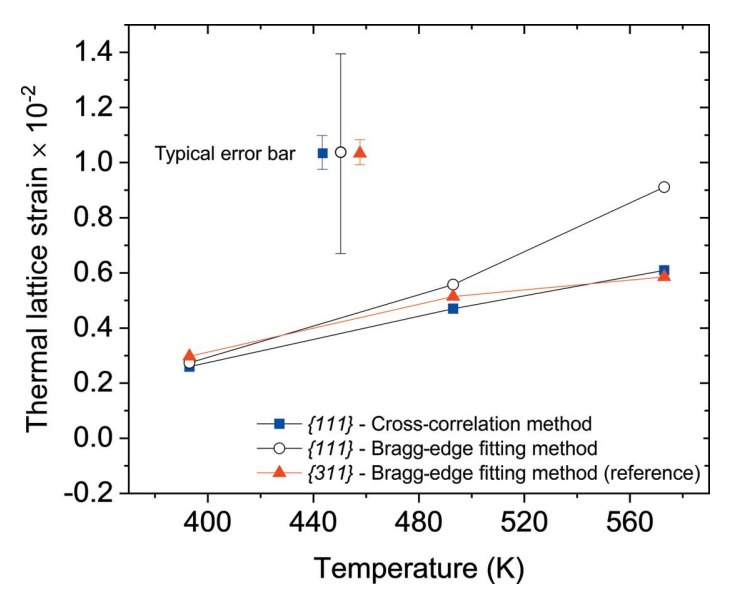

Figure 14

Thermal lattice strain of the rolled aluminium plate, analysed from the texture-affected $\mathrm{Al}$ \{111\} Bragg edge using the cross-correlation method and the Bragg-edge function-fitting method. The cross-correlation results agree well with the reference, i.e. thermal lattice strain analysed from the 'powder-like' $\mathrm{Al}\{311\}$ Bragg edge using the function-fitting method, demonstrating the capability of cross-correlation for measuring strain on textured samples. All the Bragg edges were extracted from detection-area sizes of $268 \times 268$ pixels. 
investigate how higher-order differentiation can help deal with noisy data in the cross-correlation method, and whether Bragg edges with distinctly different shapes, for example because of averaging over significant strain gradients, can be treated with the correlation method.

\section{Application of Bragg-edge transmission imaging on IMAT}

Following the characterization of the IMAT instrument and the introduction of a new analysis method in the previous section, here we present selected applications of Bragg-edge transmission imaging on IMAT. Bragg-edge transmission for residual strain mapping application is described in Section 5.1, texture mapping is presented in Section 5.2, and the use of Bragg-edge analysis for determining the temperature dependency of a Debye-Waller factor is presented in Section 5.3.

\subsection{Strain mapping}

Residual strain mapping of engineering components can be performed either using mechanical relaxation approaches such as the contour method or via non-destructive methods, such as synchrotron X-ray and neutron diffraction. In the case of diffraction-based measurements, full strain mapping requires tens to hundreds of measurement points, thus increasing the complexity in terms of measurement setup and acquisition time. The spatial resolution of those measurements is rather limited. Neutron transmission on IMAT offers the possibility to produce through-thickness $2 \mathrm{D}$ strain maps with higher spatial resolution and shorter measurement times.

To demonstrate this, residual strain mapping was performed on an $\mathrm{AlSiC}_{\mathrm{p}}$ metal matrix composite (MMC). The material is an attractive option for engineering and structural applications (El-Gallab \& Sklad, 1998). The composite sample was constituted of an Al2124 matrix and pure silicon carbide particulates at $20 \%$ weight fraction, and was fabricated by Aerospace Metal Composites, UK (now Materion AMC, UK). The production includes a heat-treatment process comprising a solution heat treatment followed by cold water quenching, which is expected to introduce a parabolic residual strain variation through the thickness of the plate. The sample dimensions are $15 \times 35 \times 35 \mathrm{~mm}$. Neutron transmission data were collected on IMAT by placing the sample $\sim 29 \mathrm{~mm}$ in front of the MCP detector. The measurement time was $4 \mathrm{~h}$ using a pinhole of $40 \mathrm{~mm}(L / D=250)$. The sample was aligned so that the measured strain is averaged in the $x$-axis direction; the coordinate system and the experimental setup are shown in Fig. 15(a). Neutron diffraction on the same sample was performed at ENGIN-X (Santisteban, Daymond et al., 2006) for comparison. A gauge volume of $2 \times 2 \times 10 \mathrm{~mm}$ was used, with 13 measurement points located along the $z$ axis and with $30 \mathrm{~min}$ measurement time per point. The gauge volume was elongated along the $y$ axis, since minimum strain variation was expected in this direction and the diffracting volume could thus be increased without compromising the strain resolution. The sample was aligned to measure the $x$-axis strain [Fig. 15(a)]. For both neutron transmission and diffraction, an
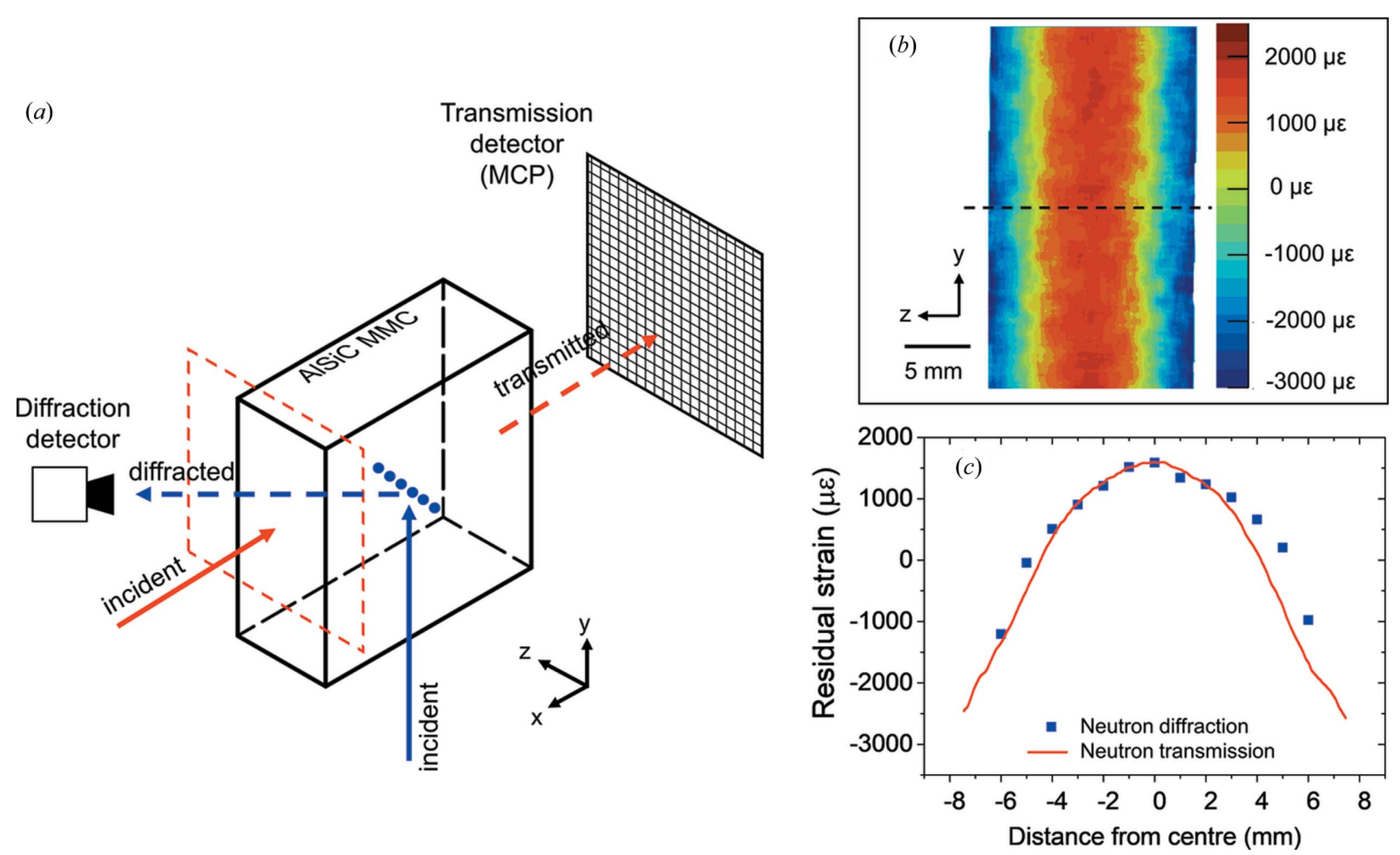

Figure 15

(a) Setup of neutron transmission (red lines and arrows) and neutron diffraction (blue dots and arrows) measurements on an $\mathrm{AlSiC}_{\mathrm{p}}$ metal matrix composite. (b) Residual strain map in the $x$ direction produced by neutron transmission, showing compressive strains at both surfaces of the sample and tensile strains in the middle of the sample. (c) Comparison between neutron transmission (red line) and neutron diffraction (blue symbols) results. 
Al powder which constitutes the composite matrix material was used for measurement of the strain-free lattice parameter $d_{0}$. The $\mathrm{Al}\{111\}$ Bragg edge was used for analysis to obtain the strain map from the transmission signal. The Bragg-edge fitting method was used, and fitting was performed using the BEATRIX software (Minniti, 2019) with a macro-pixel size of $20 \times 20$. Neutron diffraction data were analysed by fitting the Al \{111\} Bragg peak, using single-peak fitting with a doubleexponential TOF function convoluted with the Voigt function (Santisteban, Daymond et al., 2006).

A map of the $x$-axis residual strain of the $\mathrm{AlSiC}_{\mathrm{p}} \mathrm{MMC}$ sample is shown in Fig. 15(b). Compressive residual strain can be found at both surfaces of the composite. The residual strain gradually changes to tensile strain as it moves towards the middle of the sample. A parabolic shaped, symmetrical strain profile through the thickness of the plate can be clearly observed, while minimal strain variation along the $z$-axis direction can be seen. A line profile from the strain map, for the dashed line indicated in Fig. 15(b), shows that results from neutron transmission agree very well with results from neutron diffraction [Fig. 15(c)], with average differences less than $270 \mu \varepsilon$. The discrepancies are reasonable because of the difference in the gauge volumes from which the values were averaged in the two measurements. Considering the reliability of the diffraction result, this further shows the accuracy of the transmission measurement, as has been previously highlighted in this paper through the high-temperature experiment. The corresponding neutron transmission setup has a sample-todetector distance of $l=64 \mathrm{~mm}(29 \mathrm{~mm}+35 \mathrm{~mm}$ of sample thickness in the transmission direction) and $L / D=250$, and therefore has a geometrical blurring of $d=0.256 \mathrm{~mm}$. According to Fig. 9, this $d$ value and a macro-pixel size of $20 \times$ 20 yielded the spatial resolution of the reconstructed map of around $600 \mu \mathrm{m}$. This high-resolution 2D map was obtained within $4 \mathrm{~h}$ of data collection. Obtaining a map with similar resolution with neutron diffraction would require hundreds of measurement points and would take much longer.

\subsection{Crystallographic texture mapping and analysis}

Crystallographic texture plays an important role in controlling material properties. Mapping of crystallographic texture across a sample is usually performed using electron backscatter diffraction (EBSD) in a scanning electron microscope. While orientation distributions can be obtained through EBSD, the technique requires careful sample preparation and a relatively long scanning process, and only reveals the texture on the surface being measured. Texture analysis using neutron diffraction, such as on GEM at ISIS (Kockelmann et al., 2006), can provide information from the bulk sample volume. Time-of-flight neutron transmission offers the mapping of texture information through Bragg-edge analysis. An example is provided by mapping the texture distribution of a rolled Al2024-T351 plate with dimensions of $50 \times 50 \times 15 \mathrm{~mm}$. Two measurements were performed, with the rolling direction parallel and perpendicular to the neutron beam [Fig. 16(a) and 16(b), respectively]. The texture is quantified by calculating the factor $R$ [equation (5)] for a wavelength of $4.68 \AA$, which corresponds to the position of the $\mathrm{Al}\{111\}$ Bragg edge. The base attenuation, $I_{\text {base }}$, is determined by using a radiograph collected above the $\{111\}$ Bragg edge at $4.78 \AA$ [see Kockelmann et al. (2007) for details]. Therefore, the $R$-factor map displays the number of crystals having the (111) normals aligned with the direction of the incident beam.

The $R$-factor maps for a wavelength of $4.68 \AA$ are shown in Figs. 16(c) and 16(d) for the rolling direction parallel and perpendicular to the beam direction, respectively. It can be seen from Fig. 16(c) that the $R$ map exhibits a distinct pattern with $R>1$ at both surfaces of the plate and lower values $R<1$ in the middle section. The result means that at the surfaces there are a larger number of crystallites having the (111) plane normals parallel to the beam direction (and to the rolling direction), while in the middle fewer (111) planes are found with such orientation. The inverse of this $R$ map [Fig. 16(d)] is observed for the measurement direction rotated by an angle of $90^{\circ}$. Such symmetrical texture distributions through the thickness of the sample are indicative of a rolling process during manufacture, similarly shown by Sun et al. (2018) on rolled stainless steel. Since the mapping was performed without macro-pixel averaging, the spatial resolution of the map is solely determined by the geometrical blurring. Considering a value of $l=79 \mathrm{~mm}(29 \mathrm{~mm}$ of sample-todetector distance $+50 \mathrm{~mm}$ of sample thickness in the transmission direction to calculate the worst geometrical blurring), and for an $L / D$ ratio of 250 , the geometrical blurring is $d=$ $0.316 \mathrm{~mm}$. Interpolating from the values in Section 4.2 for $d=$ $0.316 \mathrm{~mm}$ yields a spatial resolution of around $130 \mu \mathrm{m}$. The spatial resolution is obviously higher for thinner samples in the transmission direction: values as low as $\sim 90 \mu \mathrm{m}$ have been shown in Section 4.2. The result demonstrates the ability of

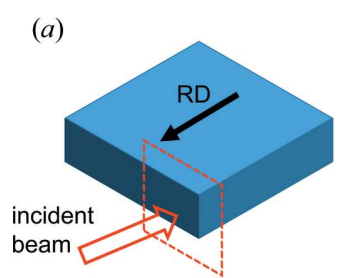

(c)

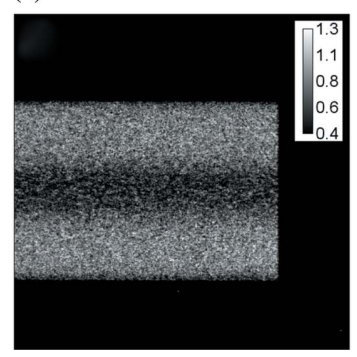

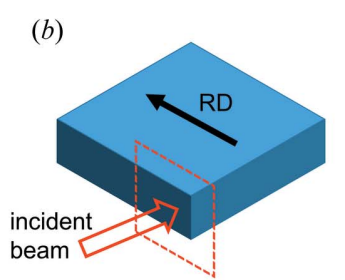

(d)

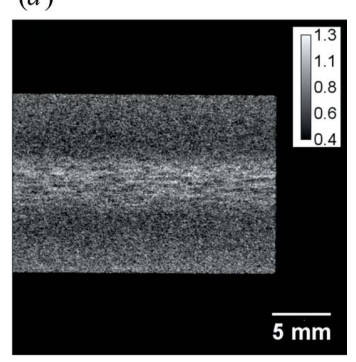

Figure 16

Neutron transmission maps of a rolled Al2024-T351 plate with rolling direction (RD) parallel $(a)$ and perpendicular $(b)$ to the incident beam. The field of view is shown by the dashed box. Map of $R$ factor (m.r.d.) for the $\{111\}$ edge at $\lambda=4.68 \AA(\beta=0)$ for measurement with RD parallel $(c)$ and perpendicular $(d)$ to the incident beam. 


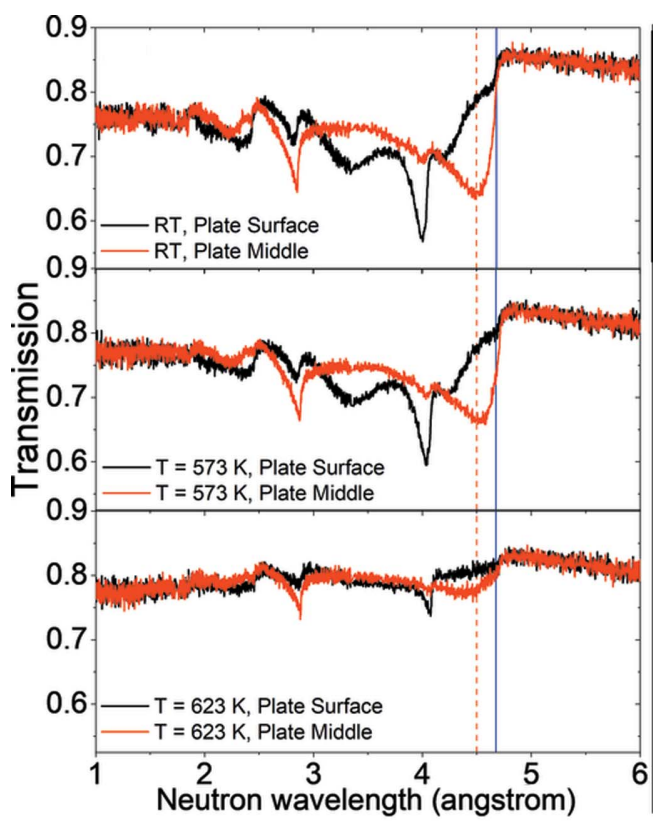

(a)

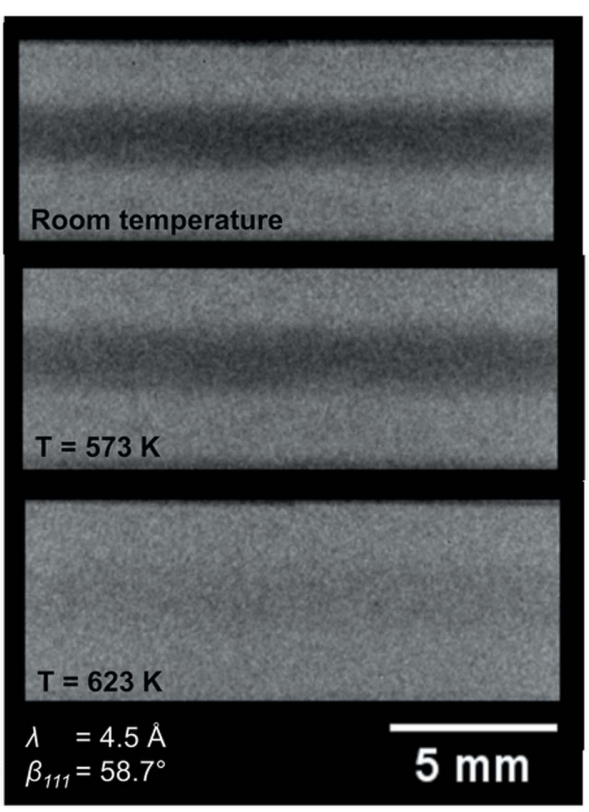

(b)

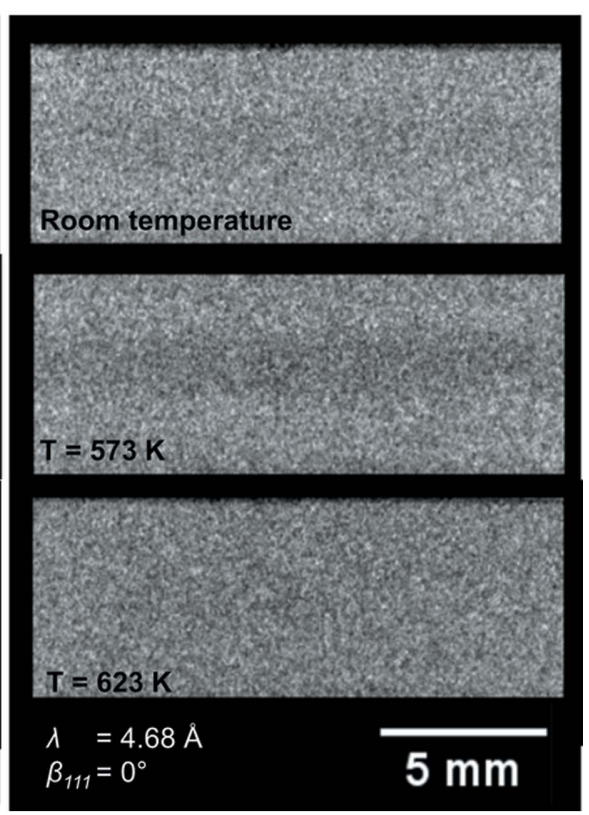

(c)

Figure 17

(a) Transmission spectra of rolled $\mathrm{Al}$ plate taken at the surface and the middle of the plate, measured at room temperature $(\mathrm{RT}), T=573 \mathrm{~K}$ and $T=$ $623 \mathrm{~K}$. Vertical solid blue and dashed red lines indicate the wavelengths for which radiographs were selected. $(b)$ Radiographs of the plate taken using a wavelength of $4.5 \AA\left(\beta_{111}=58.7^{\circ}\right)$ at room temperature and $573 \mathrm{~K}$, showing $\{111\}$ planes preferentially aligned normal to the beam in the middle section of the sample. The texture variation diminished at $623 \mathrm{~K}$ owing to recrystallization. (c) Radiographs taken at a wavelength of $4.68 \AA\left(\beta_{111}=0^{\circ}\right)$ at room temperature, $573 \mathrm{~K}$ and $623 \mathrm{~K}$, indicating a weak texture variation.

neutron transmission imaging to visualize texture distribution quickly and non-destructively.

One of the benefits of non-destructive mapping with neutrons is that texture evolution can be visualized during a process, for example during heat treatment. Revisiting the transmission from a rolled aluminium plate at room and high temperature [Fig. 17(a)], it can be observed that there are no significant changes from room temperature to $573 \mathrm{~K}$ in terms of the shape of the transmission spectra, with Bragg edges indicating the presence of significant crystallographic texture. There are clear differences between transmission spectra taken from the surface (black curve) and the middle of the plate (red curve) [Fig. 17(a)], indicating texture variation from top to bottom. Increasing the temperature further by $50 \mathrm{~K}$ (from 573 to $623 \mathrm{~K}$ ), however, changes the spectra drastically. The Bragg-edge height decreases at $623 \mathrm{~K}$, and the spectra start to resemble those expected for randomly oriented crystallites. Also, the difference between the surface and the middle section of the sample is much less apparent at that temperature. This behaviour is caused by the recrystallization of the aluminium plate. When recrystallization occurs on deformed polycrystalline materials, the newly developed grains are either randomly oriented or weakly textured (Humphreys \& Hatherly, 2004), thus explaining the change in the shape of the transmission curves. It is also possible that grain growth has started to occur for Al1050 at $623 \mathrm{~K}$, and therefore the large crystallite size might induce an extinction effect which reduces the Bragg-edge intensity (Sato et al., 2011). The recrystallization temperature of commercial aluminium alloys is $613 \mathrm{~K}$ (Hatch, 1984), which matches the temperature range where the abrupt changes in the transmission spectra were observed.

Texture variation in a sample can be made visible by energy-dispersive imaging. Radiographs taken at $4.5 \AA$, indicated by the vertical red dashed line in Fig. 17(a), showed a distinct texture distribution pattern produced by a rolling process [Fig. 17(b)]. This indicates a different number of crystallites at the surface and the middle section of the sample being preferentially aligned, with the (111) poles making an angle of $\beta_{111}=58.7^{\circ}$ with the incident neutron beam. Above the recrystallization temperature, the texture variation between the surface and the middle section of the plate is less pronounced, indicating a greater number of randomly oriented grains throughout the sample thickness. Radiographs taken at $4.68 \AA$, indicated by the vertical blue line in Fig. 17(a), were provided as comparison and do not show such variation of preferred orientation [Fig. 17(c)]. This wavelength corresponds to the $\{111\}$ Bragg-edge position. More comprehensive quantification of the texture and grain-size information can be performed using either Bragg-edge modelling, which includes structural parameters (Song et al., 2017); Rietveld-type fullpattern refinement (Sato et al., 2011); or Bragg-dip transmission imaging (Sato et al., 2017; Malamud \& Santisteban, 2016). There is scope to perform such quantitative analyses on IMAT by implementing these data analysis methods in the future.

\subsection{Debye-Waller factor analysis}

The Debye-Waller parameter approximates the thermal motion of atoms around their crystallographic positions. The 
Debye-Waller factor $\exp (-2 W)$ describes the effect of the thermal motion of atoms on the scattered intensity (Prakash $e t$ al., 1975). The exponent $2 W$ expresses this motion numerically and is directly related to the magnitude of average displacements of atoms in the crystal. When the thermal motion increases, the atoms deviate further from their original position and thus the spread of $d$ spacing increases. As the $d$ spacing changes, Bragg's law, shown in equation (2), is less well satisfied; coherent scattering is partially destroyed and the Bragg-edge height decreases [Fig. 10(a)], in the same way that Bragg-peak intensities are depressed at higher temperatures. Accordingly, it is difficult to separate the effect of the DebyeWaller factor from other effects such as magnetic scattering, extinction and crystallographic texture through single-Braggedge analysis. However, the temperature dependence of the Debye-Waller factor in a material can still be extracted. The same information has been obtained using different experimental methods such as X-ray diffraction (Haworth, 1960) and $\gamma$-ray resonant absorption (Debrunner \& Morrison, 1965). However, neutron transmission offers the possibility to map the information across a sample. As proof of concept, the temperature dependence of the Debye-Waller factor of $\alpha$-iron was quantified through Bragg-edge analysis. The data were derived from the $\mathrm{Fe}$ powder data collected at different temperatures as described in Section 3.3. Previously Haworth (1960), Prakash et al. (1975) and Kharoo et al. (1977) compared the Debye-Waller-factor dependencies on temperature in terms of the temperature parameter $Y$, which is defined by

$$
\begin{aligned}
Y & =\log _{10} \exp (\lambda / \sin \theta)^{2}\left(2 W_{T_{0}}-2 W_{T}\right) \\
& =(\lambda / \sin \theta)^{2} \log _{10}\left(\rho_{T} / \rho_{T_{0}}\right),
\end{aligned}
$$

where $2 W_{T}$ and $2 W_{T_{0}}$ are the Debye-Waller exponents for temperatures $T$ and $T_{0}$, respectively, $\rho_{T}$ and $\rho_{T_{0}}$ are the Braggedge heights for temperatures $T$ and $T_{0}$, respectively, and $\sin \theta$ is equal to 1 for transmission. The Bragg-edge height is

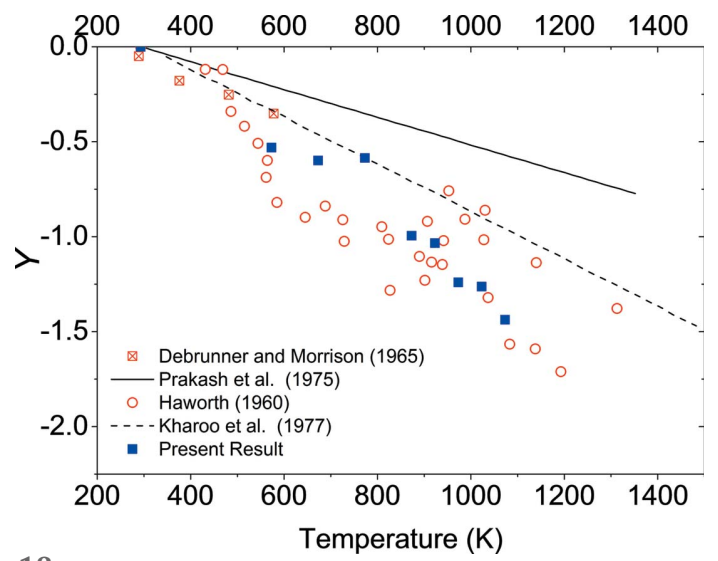

Figure 18

Temperature parameter $Y$ of $\mathrm{Fe}$ obtained at different temperatures through Bragg-edge analysis, compared with literature values measured by $\gamma$-ray resonant absorption (Debrunner \& Morrison, 1965) and X-ray diffraction (Haworth, 1960). Theoretical values from Prakash et al. (1975) and Kharoo et al. (1977) are shown for comparison as solid and dashed lines, respectively. calculated using the first term on the right-hand side of equation (3):

$$
\rho=\exp \left[-\left(a_{0}+b_{0} \lambda_{h k l}\right)\right]\left\{1-\exp \left[-\left(a_{h k l}+b_{h k l} \lambda_{h k l}\right)\right]\right\} .
$$

Room temperature was used as $T_{0}$ in both the present work and literature studies.

Fig. 18 shows the values of $Y$ for Fe at different temperatures. In the present work, the values were obtained by fitting the Fe $\{220\}$ Bragg edges, selected because they make the minimum contribution of magnetic scattering to the transmission spectra at shorter wavelengths (Powers, 1938). The present result is compared against previous experimental values from Debrunner \& Morrison (1965) as mentioned by Prakash et al. (1975) and Kharoo et al. (1977), and from Haworth (1960), who used X-ray diffraction and similarly focused on the $\mathrm{Fe}\{220\}$ diffraction peak covering the temperature range of $286-1190 \mathrm{~K}$. Theoretical values calculated by Prakash et al. (1975) and Kharoo et al. (1977) are also shown. The values from the present work agree well with the previous experimental values, considering the scatter in the results by Haworth. Meanwhile, both present and previous experimental values deviate from the theoretical calculations, especially at higher temperatures, which is explained by the inclusion of several parameters in the model (Prakash et al., 1975). Nevertheless, the results show that information on the temperature dependence of the Debye-Waller factor can be derived from neutron transmission data.

\section{Conclusions}

IMAT is a new instrument for materials imaging and strain and texture analysis, situated at the ISIS pulsed neutron source. IMAT instrument characterization, spatial resolution determination and strain validation for Bragg-edge transmission imaging has been carried out and is presented in this paper. The application of the technique for different materials analyses using the instrument has also been demonstrated. The conclusions are as follows:

(1) Accurate strain mapping can be performed on the IMAT beamline. Typical measurements on IMAT with an MCP detector can provide a quarter of a million spatially resolved measurement points in a single exposure with counting times below $10 \mathrm{~h}$. The detector has a single-pixel size of $55 \times 55 \mu \mathrm{m}$. Pixel binning is often necessary to improve the counting statistics, but a strain map can be reconstructed with a spatial resolution in the range of 300-600 $\mu \mathrm{m}$. Such measurements provide strain uncertainties below $200 \mu \varepsilon$, and uncertainties as low as $\sim 90 \mu \varepsilon$ have been obtained. These numbers show that IMAT is suitable for strain-mapping applications on engineering samples.

(2) For the first time, we have demonstrated that Braggedge analysis based on cross-correlation has provided an alternative measure of lattice-parameter shifts for both nontextured and textured samples. For non-textured samples, strain values derived from Bragg-edge fitting were successfully reproduced by the cross-correlation method. For Bragg edges from textured samples, where the analytical parameter-fitting 
approach often struggles to describe the Bragg-edge shape, the cross-correlation method was shown to provide accurate results. The counting statistics of the neutron data still limit the implementation of the method in strain-mapping software tools, but, as neutron instrument and detector technologies improve, use of the cross-correlation method for strain mapping will become feasible in the future.

(3) We have demonstrated that Bragg-edge neutron transmission has applications in several domains by offering the possibility to provide spatially resolved information on the local crystallographic structure. Mapping of residual strain inside an engineering component was successfully performed, with results showing very good agreement with neutron diffraction results. Crystallographic texture distributions and evolution can also be mapped using Bragg-edge transmission with a scope of quantifying the texture parameters with a more comprehensive model. A proof of concept of measuring temperature dependences of structure parameters, such as Debye-Waller factor, through Bragg-edge analysis has been demonstrated, with results agreeing well with previous theoretical and experimental values.

\section{Acknowledgements}

The authors are grateful for the provision of beam time at ENGIN-X, ISIS (RB1720411), and would like to thank Dr Saurabh Kabra and Dr Tung Lik Lee for support during the experiment.

\section{Funding information}

RSR is supported by a Facility Development Studentship from the UK Science and Technology Facilities Council (STFC). MEF is grateful for funding from the Lloyd's Register Foundation, a charitable foundation helping to protect life and property by supporting engineering-related education, public engagement and the application of research.

\section{References}

Abbey, B., Zhang, S. Y., Vorster, W. \& Korsunsky, A. M. (2012). Nucl. Instrum. Methods Phys. Res. B, 270, 28-35.

Basinski, Z. S., Hume-Rothery, W. \& Sutton, A. L. (1955). Proc. $R$. Soc. London Ser. A, 229, 459-467.

Boin, M. (2012). J. Appl. Cryst. 45, 603-607.

Boin, M., Hilger, A., Kardjilov, N., Zhang, S. Y., Oliver, E. C., James, J. A., Randau, C. \& Wimpory, R. C. (2011). J. Appl. Cryst. 44, 10401046.

Bourbia, A., Draissia, M., Bedboudi, H., Boulkhessaim, S. \& Debili, M. Y. (2010). J. X-ray Sci. Technol. 18, 201-219.

Burca, G., Kockelmann, W., James, J. A. \& Fitzpatrick, M. E. (2013). J. Instrum. 8, P10001.

Cao, R. L. \& Biegalski, S. R. (2007). Nucl. Instrum. Methods Phys. Res. A, 582, 621-628.

Cooper, A. S. (1962). Acta Cryst. 15, 578-582.

Davey, W. P. (1925). Phys. Rev. 25, 753-761.

Debrunner, P. \& Morrison, R. J. (1965). Rev. Sci. Instrum. 36, 145149.

El-Gallab, M. \& Sklad, M. (1998). J. Mater. Process. Technol. 83, 151158.
Figgins, B. F., Jones, G. O. \& Riley, D. P. (1956). Philos. Mag. 1, $747-$ 758.

Gorton, A. T., Bitsianes, G. \& Joseph, T. L. (1965). Trans. Met. Soc. Aime, 233, 1519-1525.

Harwood, M. G. (1949). Nature, 164, 787.

Hatch, J. E. (1984). Aluminum: Properties and Physical Metallurgy. Metals Park: American Society for Metals.

Haworth, C. W. (1960). Philos. Mag. 5, 1229-1234.

Hendriks, J. N., Gregg, A. W. T., Wensrich, C. M., Tremsin, A. S., Shinohara, T., Meylan, M., Kisi, E. H., Luzin, V. \& Kirsten, O. (2017). Phys. Rev. Mater. 1, 053802.

Hull, A. W. (1917). Phys. Rev. 10, 661-696.

Humphreys, F. J. \& Hatherly, M. (2004). Recrystallization and Related Annealing Phenomena, pp. 379-413. Oxford: Elsevier.

Itoh, T., Mori, M., Inukai, M., Nitani, H., Yamamoto, T., Miyanaga, T., Igawa, N., Kitamura, N., Ishida, N. \& Idemoto, Y. (2015). J. Phys. Chem. C, 119, 8447-8458.

Jette, E. R. \& Foote, F. (1935). J. Chem. Phys. 3, 605-616.

Kharoo, H. L., Gupta, O. P. \& P. Hemkar, M. (1977). J. Phys. Soc. Jpn, 43, 2030-2035.

Kochanovska, A. (1949). Physica, 15, 191-196.

Kockelmann, W., Chapon, L. C. \& Radaelli, P. G. (2006). Phys. B Condens. Matter. 385-386, 639-643.

Kockelmann, W., Frei, G., Lehmann, E. H., Vontobel, P. \& Santisteban, J. R. (2007). Nucl. Instrum. Methods Phys. Res. A, 578, 421-434.

Kockelmann, W., Minniti, T., Pooley, D., Burca, G., Ramadhan, R., Akeroyd, F., Howells, G., Moreton-Smith, C., Keymer, D., Kelleher, J., Kabra, S., Lee, T., Ziesche, R., Reid, A., Vitucci, G., Gorini, G., Micieli, D., Agostino, R., Formoso, V., Aliotta, F., Ponterio, R., Trusso, S., Salvato, G., Vasi, C., Grazzi, F., Watanabe, K., Lee, J., Tremsin, A., McPhate, J., Nixon, D., Draper, N., Halcrow, W. \& Nightingale, J. (2018). J. Imaging, 4, 47.

Kockelmann, W., Zhang, S. Y., Kelleher, J. F., Nightingale, J. B., Burca, G. \& James, J. A. (2013). Phys. Proc 43, 100-110.

Kümmerle, E. A. \& Heger, G. (1999). J. Solid State Chem. 147, 485500.

Makowska, M. G., Strobl, M., Lauridsen, E. M., Kabra, S., Kockelmann, W., Tremsin, A., Frandsen, H. L. \& Theil Kuhn, L. (2016). J. Appl. Cryst. 49, 1674-1681.

Malamud, F. \& Santisteban, J. R. (2016). J. Appl. Cryst. 49, 348-365.

Malamud, F., Santisteban, J. R., Vicente Alvarez, M. A., Bolmaro, R., Kelleher, J., Kabra, S. \& Kockelmann, W. (2014). J. Appl. Cryst. 47, 1337-1354.

Minniti, T. (2019). J. Appl. Cryst. Submitted.

Minniti, T., Kockelmann, W., Burca, G., Kelleher, J. F., Kabra, S., Zhang, S. Y., Pooley, D. E., Schooneveld, E. M., Mutamba, Q., Sykora, J., Rhodes, N. J., Pouzols, F. M., Nightingale, J. B., Aliotta, F., Bonaccorsi, L. M., Ponterio, R., Salvato, G., Trusso, S., Vasi, C., Tremsin, A. S. \& Gorini, G. (2016). J. Instrum. 11, C03014.

Minniti, T., Watanabe, K., Burca, G., Pooley, D. E. \& Kockelmann, W. (2018). Nucl. Instrum. Methods Phys. Res. A, 888, 184-195.

Owen, E. A. \& Yates, E. L. (1933). Philos. Mag. 7, 1933.

Pooley, D. E., Lee, J. W. L., Brouard, M., John, J. J., Kockelmann, W., Rhodes, N. J., Schooneveld, E. M., Sedgwick, I., Turchetta, R. \& Vallance, C. (2017). IEEE Trans. Nucl. Sci. 64, 2970-2981.

Powers, P. N. (1938). Phys. Rev. 54, 827-838.

Prakash, J., Pathak, L. P. \& Hemkar, M. P. (1975). Aust. J. Phys. 28, 63-68.

Ramadhan, R. S., Syed, A. K., Tremsin, A. S., Kockelmann, W., Dalgliesh, R., Chen, B., Parfitt, D. \& Fitzpatrick, M. E. (2018). Mater. Des. 143, 56-64.

Santisteban, J. R., Daymond, M. R., James, J. A. \& Edwards, L. (2006). J. Appl. Cryst. 39, 812-825.

Santisteban, J. R., Edwards, L. \& Stelmukh, V. (2006). Phys. B Condens. Matter. 385-386, 636-638.

Santisteban, J. R., Edwards, L., Steuwer, A. \& Withers, P. J. (2001). J. Appl. Cryst. 34, 289-297. 
Sato, H., Kamiyama, T. \& Kiyanagi, Y. (2011). Mater. Trans. 52, $1294-$ 1302.

Sato, H., Sato, T., Shiota, Y., Kamiyama, T., Tremsin, A. S., Ohnuma, M. \& Kiyanagi, Y. (2015). Mater. Trans. 56, 1147-1152.

Sato, H., Shiota, Y., Morooka, S., Todaka, Y., Adachi, N., Sadamatsu, S., Oikawa, K., Harada, M., Zhang, S., Su, Y., Kamiyama, T., Ohnuma, M., Furusaka, M., Shinohara, T. \& Kiyanagi, Y. (2017). J. Appl. Cryst. 50, 1601-1610.

Sato, H., Shiota, Y., Shinohara, T., Kamiyama, T., Ohnuma, M., Furusaka, M. \& Kiyanagi, Y. (2015). Phys. Proc. 69, 349-357.

Song, G., Lin, J., Bilheux, J., Xie, Q., Santodonato, L., Molaison, J., Skorpenske, H. M., Dos Santos, A., Tulk, C., An, K., Stoica, A., Kirka, M., Dehoff, R., Tremsin, A., Bunn, J., Sochalski-Kolbus, L. \& Bilheux, H. (2017). J. Imaging, 3, 65.

Stecura, S. \& Campbell, W. J. (1961). Thermal Expansion and Phase Inversion of Rare-Earth Oxides. Washington, DC: US Department of the Interior, Bureau of Mines.

Straumanis, M. E. \& Kim, D. C. (1969). Z. Metallkd. 60, 272-277.

Strobl, M., Woracek, R., Kardjilov, N., Hilger, A., Wimpory, R., Tremsin, A., Wilpert, T., Schulz, C., Manke, I. \& Penumadu, D. (2012). Nucl. Instrum. Methods Phys. Res. A, 680, 27-34.

Sun, T., Tremsin, A. S., Roy, M. J., Hofmann, M., Prangnell, P. B. \& Withers, P. J. (2018). Mater. Sci. Eng. A, 712, 531-538.

Swanson, H. E. \& Tatge, E. (1953). Standard X-ray Diffraction Powder Patterns, Circular of the Bureau of Standards No. 539, Vol. 1, pp. 1-95. National Bureau of Standards.

Treimer, W. (2009). Neutron Imaging and Applications: A Reference for the Imaging Community, edited by I. S. Anderson, R. L. Mc Greevy \& H. Z. Bilheux, pp. 81-108. New York: Springer.

Tremsin, A. S., Ganguly, S., Meco, S. M., Pardal, G. R., Shinohara, T. \& Feller, W. B. (2016). J. Appl. Cryst. 49, 1130-1140.
Tremsin, A., Kockelmann, W., Kelleher, J., Paradowska, A., Ramadhan, R. \& Fitzpatrick, M. (2018). J. Imaging, 4, 48.

Tremsin, A. S., McPhate, J. B., Kockelmann, W. A., Vallerga, J. V., Siegmund, O. H. W. \& Feller, W. B. (2009). IEEE Trans. Nucl. Sci. 56, 2931-2937.

Tremsin, A. S., Vallerga, J. V., McPhate, J. B. \& Siegmund, O. H. W. (2015). Nucl. Instrum. Methods Phys. Res. A, 787, 20-25.

Tremsin, A. S., Vallerga, J. V., McPhate, J. B., Siegmund, O. H. W. \& Raffanti, R. (2013). IEEE Trans. Nucl. Sci. 60, 578-585.

Tremsin, A. S., Yau, T. Y. \& Kockelmann, W. (2016). Strain, 52, 548558.

Varez, A., Garcia-Gonzalez, E., Jolly, J. \& Sanz, J. (2007). J. Eur. Ceram. Soc. 27, 3677-3682.

Vitucci, G., Minniti, T., Di Martino, D., Musa, M., Gori, L., Micieli, D., Kockelmann, W., Watanabe, K., Tremsin, A. S. \& Gorini, G. (2018). Microchem. J. 137, 473-479.

Vogel, S. (2000). Dissertation, Christian-Albrechts-Universität zu Kiel, Germany.

Whitfield, H. J., Roman, D. \& Palmer, A. R. (1966). J. Inorg. Nucl. Chem. 28, 2817-2825.

Wilson, A. J. C. (1941). Proc. Phys. Soc. 53, 235-244.

Wołcyrz, M. \& Kepinski, L. (1992). J. Solid State Chem. 99, 409413.

Woracek, R., Penumadu, D., Kardjilov, N., Hilger, A., Boin, M., Banhart, J. \& Manke, I. (2015). Phys. Proc. 69, 227-236.

Woracek, R., Penumadu, D., Kardjilov, N., Hilger, A., Strobl, M., Wimpory, R. C., Manke, I. \& Banhart, J. (2011). J. Appl. Phys. 109, 2-6.

Woracek, R., Santisteban, J., Fedrigo, A. \& Strobl, M. (2018). Nucl. Instrum. Methods Phys. Res. A, 878, 141-158.

Yang, Y. W. \& Coppens, P. (1978). Acta Cryst. A34, 61-65. 